\title{
Highly Enantioselective Access to Primary Propargylamines: 4-Piperidone as a Convenient Protecting Group
}

\author{
Patrick Aschwanden, Corey R. J. Stephenson and Erick M. Carreira*
}

The following includes general experimental procedures, specific details for representative reactions, and isolation and spectroscopic information for the new compounds prepared. All reactions were performed in oven dried glassware under argon. ${ }^{1} \mathrm{H}$ and ${ }^{13} \mathrm{C}$ NMR spectra were recorded on a VARIAN Mercury $300 \mathrm{MHz}$ or a Gemini 300 MHz. Infrared spectra were recorded on a Perkin-Elmer RX-I FT-IR. High resolution mass spectra were obtained on a VG-TRIBRID for electron impact ionization (EI) and Ion spec Ultima 4.7 spectrometer for MALDI. Optical rotations $[\alpha]_{D}$ were measured on a Jasco DIP-1000 Polarimeter.

\section{General Procedure 1 (GP 1)}

A 10-mL Schlenk tube was charged with $\mathrm{CuBr}$ ( $3.6 \mathrm{mg}, 0.025 \mathrm{mmol}, 0.050$ equiv), ligand $1(15 \mathrm{mg}, 0.028 \mathrm{mmol}, 0.055$ equiv) and $0.25 \mathrm{~g}$ powedered $4 \AA$ molecular sieves and purged with argon. To the flask was added $1 \mathrm{~mL}$ of $\mathrm{CH}_{2} \mathrm{Cl}_{2}$ and the resulting suspension was stirred for 60 minutes at $23{ }^{\circ} \mathrm{C}$. To the reaction mixture was added triethylamine (56 $\mathrm{mg}, 0.55 \mathrm{mmol}, 1.1$ equiv), powdered 4-piperidone hydrochloride monohydrate $(77 \mathrm{mg}$, $0.50 \mathrm{mmol}, 1.0$ equiv), alkyne (1.0 mmol, 2.0 equiv) and freshly distilled aldehyde ( 0.50 mmol, 1.0 equiv). After addition of an additional $1 \mathrm{~mL}$ of $\mathrm{CH}_{2} \mathrm{Cl}_{2}$ the Schlenk tube was tightly sealed and the reaction was stirred at $23{ }^{\circ} \mathrm{C}$. Upon completion, the reaction was quenched by the addition of a saturated aqueous ammonium chloride solution $(3 \mathrm{~mL})$. The reaction mixture was poured into a separatory funnel containing dichloromethane (10 $\mathrm{mL}$ ). The layers were separated and the aqueous layer was extracted with dichloromethane $(3 \times 10 \mathrm{~mL})$. The combined organic layers were washed with brine (10 $\mathrm{mL}$ ), dried over $\mathrm{Na}_{2} \mathrm{SO}_{4}$ and concentrated in vacuo. Purification of the crude material by flash column chromatography on silica gel using hexane/ethyl acetate $=8: 2$ as eluent afforded the analytically pure propargyl amines. 


\section{(S)-1-(1-(Trimethylsilyl)oct-1-yn-3-yl)piperidin-4-one (2a)}

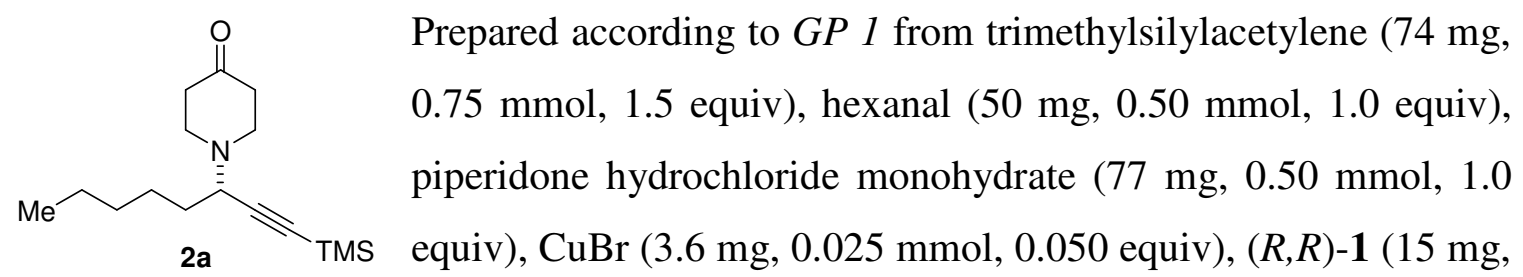
$0.028 \mathrm{mmol}, 0.055$ equiv), triethylamine (56 mg, $0.55 \mathrm{mmol}, 1.1$ equiv) and $4 \AA$ molecular sieves $(0.25 \mathrm{~g})$ in $2 \mathrm{~mL}$ dichloromethane at $23{ }^{\circ} \mathrm{C}$ for 22 hours. Purification by flash column chromatography on silica gel afforded $102 \mathrm{mg}$ (73\%) of the title compound as a colorless oil. The enantioselectivity was $90 \%$ ee as determined by GC analysis of the desilylated alkyne $\left(\mathrm{K}_{2} \mathrm{CO}_{3}, \mathrm{MeOH}\right)$ (Supelco Gamma Dex 120, $30 \mathrm{~m}$ x 0.25 mm x 0.25 $\mu \mathrm{m}$ film thickness, $115^{\circ} \mathrm{C}, \mathrm{t}_{r} 70.5 \mathrm{~min}$ (minor), $71.6 \mathrm{~min}$ (major)).

$[\alpha]_{D}^{25}=-11.0\left(c 1.2, \mathrm{CHCl}_{3}\right)$

${ }^{1} \mathbf{H}$ NMR $\left(300 \mathrm{MHz}, \mathrm{CDCl}_{3}\right) \delta 3.43(\mathrm{t}, J=7.5 \mathrm{~Hz}, 1 \mathrm{H}), 2.92-2.84(\mathrm{~m}, 2 \mathrm{H}), 2.73-2.64$ (m, 2 H), 2.53-2.37 (m, 4 H), 1.67-1.59 (m, 2 H), 1.52-1.38 (m, 2 H), 1.37-1.32 (m, 4 H), 0.89 (t, $J=7.0 \mathrm{~Hz}, 3 \mathrm{H}), 0.134(\mathrm{~s}, 9 \mathrm{H})$;

${ }^{13} \mathrm{C}$ NMR $\left(75 \mathrm{MHz}, \mathrm{CDCl}_{3}\right) \delta 209.0,103.0,90.0,57.4,49.1,41.5,33.6,31.5,26.2,22.6$, 14.1, 0.3;

FTIR (thin film) v 2957, 2933, 2860, 2815, 2160, 1723, 1469, 1409, 1337, 1249, 1205, $1133,1075 \mathrm{~cm}^{-1}$;

HRMS (EI) $m / z$ calculated for $\mathrm{C}_{16} \mathrm{H}_{28} \mathrm{NOSi}\left([\mathrm{M}-\mathrm{H}]^{+}\right)$278.1935, found 278.1936. 


\section{(R)-1-(Methyl-1-(trimethylsilyl)pent-1-yn-3-yl)piperidin-4-one (2b)}

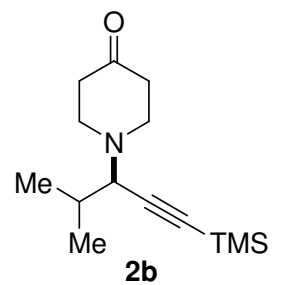

Prepared according to GP 1 from trimethylsilylacetylene (74 $\mathrm{mg}, 0.75$ mmol, 1.5 equiv), 2-methylpropanal (72 mg, $1.0 \mathrm{mmol}, 2.0$ equiv), piperidone hydrochloride monohydrate $(77 \mathrm{mg}, 0.50 \mathrm{mmol}, 1.0$ equiv), CuBr (3.6 mg, $0.025 \mathrm{mmol}, 0.050$ equiv), $(S, S)-1$ (15 mg, $0.028 \mathrm{mmol}$, 0.055 equiv), triethylamine (56 mg, $0.55 \mathrm{mmol}, 1.1$ equiv) and $4 \AA$ molecular sieves $(0.25 \mathrm{~g})$ in $2 \mathrm{~mL}$ dichloromethane at $23{ }^{\circ} \mathrm{C}$ for 22 hours. Purification by flash column chromatography on silica gel afforded $104 \mathrm{mg}(83 \%)$ of the title compound as a colorless oil. The enantioselectivity was $95 \%$ ee as determined by GC analysis of the desilylated alkyne $\left(\mathrm{K}_{2} \mathrm{CO}_{3}, \mathrm{MeOH}\right)($ Supelco Gamma Dex 120, $30 \mathrm{~m}$ x $0.25 \mathrm{~mm}$ x 0.25 $\mu \mathrm{m}$ film thickness, $120^{\circ} \mathrm{C}, \mathrm{t}_{r} 15.9 \mathrm{~min}$ (major), $16.5 \mathrm{~min}$ (minor)).

$[\alpha]_{D}^{26}=+23.9\left(c 0.6, \mathrm{CHCl}_{3}\right)$

\section{(S)-1-(Methyl-1-(trimethylsilyl)pent-1-yn-3-yl)piperidin-4-one (ent-2b)}

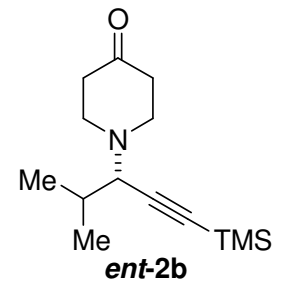

Prepared according to GP 1 from trimethylsilylacetylene (74 mg, 0.75 mmol, 1.5 equiv), 2-methylpropanal (72 mg, $1.0 \mathrm{mmol}, 2.0$ equiv), piperidone hydrochloride monohydrate $(77 \mathrm{mg}, 0.50 \mathrm{mmol}, 1.0$ equiv), CuBr (3.6 mg, $0.025 \mathrm{mmol}, 0.050$ equiv), $(R, R)-1$ (15 mg, $0.028 \mathrm{mmol}$, 0.055 equiv), triethylamine (56 mg, $0.55 \mathrm{mmol}, 1.1$ equiv) and $4 \AA$ molecular sieves $(0.25 \mathrm{~g})$ in $2 \mathrm{~mL}$ dichloromethane at $23{ }^{\circ} \mathrm{C}$ for 22 hours. Purification by flash column chromatography on silica gel afforded $111 \mathrm{mg}(88 \%)$ of the title compound as a colorless oil. The enantioselectivity was $96 \%$ ee as determined by GC analysis of the desilylated alkyne $\left(\mathrm{K}_{2} \mathrm{CO}_{3}, \mathrm{MeOH}\right)$ (Supelco Gamma Dex 120, $30 \mathrm{~m}$ x 0.25 mm x 0.25 $\mu \mathrm{m}$ film thickness, $120{ }^{\circ} \mathrm{C}, \mathrm{t}_{r} 15.8 \mathrm{~min}$ (minor), $16.2 \mathrm{~min}$ (major)).

$[\alpha]_{D}^{23}=-25.1\left(c 1.3, \mathrm{CHCl}_{3}\right)$ 
${ }^{1} \mathbf{H}$ NMR $\left(300 \mathrm{MHz}, \mathrm{CDCl}_{3}\right) \delta 2.95(\mathrm{~d}, J=10.1 \mathrm{~Hz}, 1 \mathrm{H}), 2.90-2.82(\mathrm{~m}, 2 \mathrm{H}), 2.69-2.61$ (m, 2 H), 2.53-2.37 (m, 4 H), 1.85-1.73 (m, 1 H), 1.03 (dd, $J=16.3,6.6$ Hz, 6 H), 0.15 (s, $9 \mathrm{H})$;

${ }^{13}$ C NMR (75 MHz, $\left.\mathrm{CDCl}_{3}\right) \delta 209.3,102.4,90.5,64.6,49.3,41.6,30.9,20.6,19.8,0.3$;

FTIR (thin film) v 2959, 2814, 2160, 1720, 1468, 1380, 1334, 1249, 1212, 1074, 1008 $\mathrm{cm}^{-1}$;

HRMS (EI) $m / z$ calculated for $\mathrm{C}_{11} \mathrm{H}_{18} \mathrm{NOSi}\left(\left[\mathrm{M}-\mathrm{C}_{3} \mathrm{H}_{7}\right]^{+}\right)$208.1153, found 208.1155.

\section{(S)-1-(4-Ethyl-1-(trimethylsilyl)hex-1-yn-3-yl)piperidin-4-one (2c)}

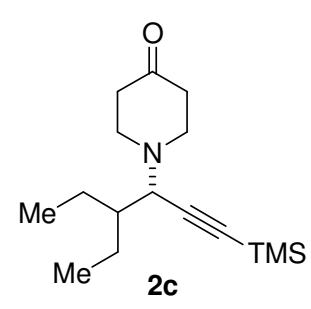

Prepared according to GP 1 from trimethylsilylacetylene $(74 \mathrm{mg}$, $0.75 \mathrm{mmol}, 1.5$ equiv), 2-ethylbutanal (100 mg, $0.50 \mathrm{mmol}, 1.0$ equiv), piperidone hydrochloride monohydrate $(77 \mathrm{mg}, 0.50 \mathrm{mmol}$, 1.0 equiv), $\mathrm{CuBr}$ (3.6 mg, $0.025 \mathrm{mmol}, 0.050$ equiv), $(R, R)-\mathbf{1}(15 \mathrm{mg}$, $0.028 \mathrm{mmol}, 0.055$ equiv), triethylamine (56 $\mathrm{mg}, 0.55 \mathrm{mmol}, 1.1$ equiv) and $4 \AA$ molecular sieves $(0.25 \mathrm{~g})$ in $2 \mathrm{~mL}$ dichloromethane at $23{ }^{\circ} \mathrm{C}$ for 22 hours. Purification by flash column chromatography on silica gel afforded $115 \mathrm{mg}(82 \%)$ of the title compound as a colorless oil. The enantioselectivity was $95 \%$ ee as determined by GC analysis of the desilylated alkyne $\left(\mathrm{K}_{2} \mathrm{CO}_{3}, \mathrm{MeOH}\right)$ (Supelco Gamma Dex 120, $30 \mathrm{~m}$ x $0.25 \mathrm{~mm}$ x $0.25 \mu \mathrm{m}$ film thickness, $115^{\circ} \mathrm{C}, \mathrm{t}_{r} 18.2$ (minor), 18.6 (major)).

$[\alpha]_{D}^{25}=-28.2\left(c 1.4, \mathrm{CHCl}_{3}\right)$

${ }^{1} \mathbf{H}$ NMR $\left(300 \mathrm{MHz}, \mathrm{CDCl}_{3}\right) \delta 3.18(\mathrm{~d}, J=9.9 \mathrm{~Hz}, 1 \mathrm{H}), 2.88-2.80$ (m, $\left.2 \mathrm{H}\right), 2.66-2.58$ (m, $2 \mathrm{H}), 1.72-1.30$ (m, $5 \mathrm{H}), 0.84$ (dt, $J=16.4,7.4 \mathrm{~Hz}, 6 \mathrm{H}), 0.12$ (s, $9 \mathrm{H})$;

${ }^{13}$ C NMR $\left(75 \mathrm{MHz}, \mathrm{CDCl}_{3}\right) \delta 209.2,102.5,90.4,60.4,49.3,41.9,41.6,22.2,20.8,10.7$, 9.7, 0.3; 
FTIR (thin film) v 2962, 2815, 2159, 1721, 1460, 1409, 1380, 1336, 1249, 1207, 1123 $\mathrm{cm}^{-1}$;

HRMS (EI) $m / z$ calculated for $\mathrm{C}_{16} \mathrm{H}_{29} \mathrm{NOSi}\left([\mathrm{M}]^{+}\right)$279.2018, found 279.2020

\section{(R)-1-(1-(Furan-2-yl)-3-(trimethylsilyl)prop-2-ynyl)piperidin-4-one (2d)}

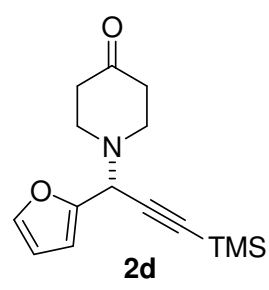

Prepared according to GP 1 from trimethylsilylacetylene (74 mg, 0.75 mmol, 1.5 equiv), furan-2-carboxaldehyde ( $48 \mathrm{mg}, 0.50 \mathrm{mmol}, 1.0$ equiv), piperidone hydrochloride monohydrate $(77 \mathrm{mg}, 0.50 \mathrm{mmol}, 1.0$ equiv), $\mathrm{CuBr}$ (3.6 mg, $0.025 \mathrm{mmol}, 0.050$ equiv), $(R, R)-\mathbf{1}(15 \mathrm{mg}$, $0.028 \mathrm{mmol}, 0.055$ equiv), triethylamine (56 $\mathrm{mg}, 0.55 \mathrm{mmol}, 1.1$ equiv) and $4 \AA$ molecular sieves $(0.25 \mathrm{~g})$ in $2 \mathrm{~mL}$ dichloromethane at $23{ }^{\circ} \mathrm{C}$ for 36 hours. Purification by flash column chromatography on silica gel afforded $80 \mathrm{mg}(58 \%)$ of the title compound as a yellowish soild: $\mathrm{mp} 52{ }^{\circ} \mathrm{C}$. The enantioselectivity was $90 \%$ ee as determined by GC analysis (Supelco Gamma Dex 120, $30 \mathrm{~m}$ x $0.25 \mathrm{~mm}$ x $0.25 \mu \mathrm{m}$ film thickness, $115^{\circ} \mathrm{C}, \mathrm{t}_{r} 97.0$ (minor), 98.6 (major)).

$[\alpha]_{D}^{25}=-2.5\left(c 1.4, \mathrm{CHCl}_{3}\right)$

${ }^{1}$ H NMR $\left(300 \mathrm{MHz}, \mathrm{CDCl}_{3}\right) \delta$ 7.38-7.39 (m, $\left.1 \mathrm{H}\right), 6.41-6.42(\mathrm{~m}, 1 \mathrm{H}), 6.31-6.32(\mathrm{~m}, 1$ H), 4.81 (s, $1 \mathrm{H}), 2.87-2.73$ (m, $4 \mathrm{H}), 2.54-2.38$ (m, 4 H), 0.17 (s, 9 H);

${ }^{13} \mathbf{C}$ NMR $\left(75 \mathrm{MHz}, \mathrm{CDCl}_{3}\right) \delta 208.4,150.7,142.8,110.0,109.6,98.1,91.9,55.5,49.0$, 41.3, 0.1;

FTIR (thin film) v 3117, 2959, 2817, 2166, 1718, 1500, 1411, 1330, 1250, 1204, 1069 $\mathrm{cm}^{-1}$;

HRMS (EI) $\mathrm{m} / z$ calculated for $\mathrm{C}_{15} \mathrm{H}_{21} \mathrm{NO}_{2} \mathrm{Si}\left([\mathrm{M}]^{+}\right)$275.1337, found 275.1335 . 


\section{(R)-1-(1-Cyclohexyl-3-(trimethylsilyl)prop-2-ynyl)piperidin-4-one (2e)}

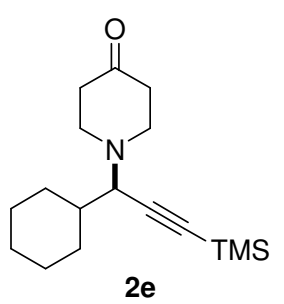

Prepared according to GP 1 from trimethylsilylacetylene $(0.15 \mathrm{~g}, 1.5$ mmol, 1.5 equiv), cyclohexanecarboxaldehyde (0.12 g, $1.0 \mathrm{mmol}, 1.0$ equiv), piperidone hydrochloride monohydrate $(0.15 \mathrm{~g}, 1.0 \mathrm{mmol}, 1.0$ equiv), $\mathrm{CuBr}$ (7.2 mg, $0.050 \mathrm{mmol}, 0.050$ equiv), $(S, S)-\mathbf{1}(31 \mathrm{mg}$, $0.055 \mathrm{mmol}, 0.055$ equiv), triethylamine $(0.11 \mathrm{~g}, 1.1 \mathrm{mmol}, 1.1$ equiv) and $4 \AA$ molecular sieves $(0.50 \mathrm{~g})$ in $4 \mathrm{~mL}$ dichloromethane at $23{ }^{\circ} \mathrm{C}$ for 22 hours. Purification by flash column chromatography on silica gel afforded $0.22 \mathrm{~g}(75 \%)$ of the title compound as a colorless foam. The enantioselectivity was $93 \%$ ee as determined by GC analysis of the desilylated alkyne $\left(\mathrm{K}_{2} \mathrm{CO}_{3}, \mathrm{MeOH}\right)$ (Supelco Gamma Dex 120, $30 \mathrm{~m}$ x $0.25 \mathrm{~mm}$ x $0.25 \mu \mathrm{m}$ film thickness, $100-140{ }^{\circ} \mathrm{C}\left(1{ }^{\circ} \mathrm{C} / \mathrm{min} \mathrm{ramp}\right)$ then hold for 45 minutes, $\mathrm{t}_{r} 72.9$ (major), 73.9 (minor)).

$[\alpha]_{D}^{25}=+15.6\left(c 0.6, \mathrm{CHCl}_{3}\right)$

${ }^{1} \mathbf{H}$ NMR $\left(300 \mathrm{MHz}, \mathrm{CDCl}_{3}\right) \delta 3.04(\mathrm{~d}, J=10.2 \mathrm{~Hz}, 1 \mathrm{H}), 2.87-2.79(\mathrm{~m}, 2 \mathrm{H}), 2.64-2.56$ (m, 2 H), 2.49-2.33 (m, 4 H), 2.03-1.99 (m, 2 H), 1.75-1.63 (m, 3 H), 1.53-1.40 (m, 1 H), 1.31-1.06 (m, $3 \mathrm{H}), 1.05-0.81$ (m, $2 \mathrm{H}), 0.11$ (s, $9 \mathrm{H})$;

${ }^{13}$ C NMR $\left(75 \mathrm{MHz}, \mathrm{CDCl}_{3}\right) \delta 209.0,102.1,90.4,63.2,49.2,41.5,39.8,31.0,30.3,26.7$, $26.1,25.9,0.3$;

FTIR (neat) v 2924, 2852, 2815, 2160, 1720, 1450, 1249, $1003 \mathrm{~cm}^{-1}$;

HRMS (EI) $m / z$ calculated for $\mathrm{C}_{16} \mathrm{H}_{26} \mathrm{NOSi}\left(\left[\mathrm{M}-\mathrm{CH}_{3}\right]^{+}\right)$276.1778, found 276.1784. 


\section{(R)-1-(1-Cyclopropyl-3-(trimethylsilyl)prop-2-ynyl)piperidin-4-one (2f)}

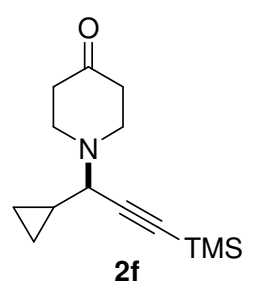

Prepared according to GP 1 from trimethylsilylacetylene $(0.48 \mathrm{~g}, 4.9$ mmol, 1.5 equiv), cyclopropanecarboxaldehyde ( $0.46 \mathrm{~g}, 6.5 \mathrm{mmol}, 2.0$ equiv), piperidone hydrochloride monohydrate $(0.50 \mathrm{~g}, 3.2 \mathrm{mmol}, 1.0$ equiv), $\mathrm{CuBr}$ (23 mg, $0.025 \mathrm{mmol}, 0.050$ equiv), (S,S)-1 (0.10 g, 0.18 mmol, 0.055 equiv), triethylamine ( $0.36 \mathrm{~g}, 3.5 \mathrm{mmol}, 1.1$ equiv) and $4 \AA$ molecular sieves $(1.6 \mathrm{~g})$ in $20 \mathrm{~mL}$ dichloromethane at $23{ }^{\circ} \mathrm{C}$ for 22 hours. Purification by flash column chromatography on silica gel afforded $0.75 \mathrm{~g}(92 \%)$ of the title compound as a colorless oil. The enantioselectivity was $89 \%$ ee as determined by ${ }^{1} \mathrm{H}$ NMR after conversion to the $(R)$-Mosher amide of $9 f\left\{{ }^{\mathbf{1}} \mathbf{H} \mathbf{~ N M R}\left(300 \mathrm{MHz}, \mathrm{CDCl}_{3}\right.\right.$, representative peaks) $\delta 3.48$ (major) (q, $J=1.5 \mathrm{~Hz}, 3 \mathrm{H}, \mathrm{OCH}_{3}$ ), 3.43 (minor) (d, $J=1.5 \mathrm{~Hz}, 1 \mathrm{H}$, $\mathrm{OCH}_{3}$ ), 2.27 (major) (d, $J=2.4 \mathrm{~Hz}, 1 \mathrm{H}$, alkyne C-H) 2.25 (minor) (d, $J=2.4 \mathrm{~Hz}, 1 \mathrm{H}$, alkyne $\mathrm{C}-\mathrm{H})\}$.

$[\alpha]_{D}^{25}=-39.4\left(c 0.8, \mathrm{CHCl}_{3}\right)$

${ }^{1} \mathbf{H}$ NMR $\left(300 \mathrm{MHz}, \mathrm{CDCl}_{3}\right) \delta 3.64(\mathrm{~d}, J=4.8 \mathrm{~Hz}, 1 \mathrm{H}), 3.10-3.01(\mathrm{~m}, 2 \mathrm{H}), 2.81-2.72$ (m, 2 H), 2.58-2.43 (m, 4 H), 1.11-1.01 (m, 1 H), 0.62-0.44 (m, 3 H), 0.41-0.33 (m, $1 \mathrm{H})$, $0.16(\mathrm{~s}, 9 \mathrm{H})$;

${ }^{13}$ C NMR $\left(75 \mathrm{MHz}, \mathrm{CDCl}_{3}\right) \delta 209.1,99.5,91.0,60.2,49.3,41.4,12.3,2.8,1.6,0.04$;

FTIR (neat) v 2960, 2902, 2815, 2162, 1720, 1420, 1250, 1210, 1121, $1075 \mathrm{~cm}^{-1}$;

HRMS (EI) $m / z$ calculated for $\mathrm{C}_{14} \mathrm{H}_{23} \mathrm{NOSi}\left([\mathrm{M}]^{+}\right)$249.1544, found 249.1550. 


\section{(R)-1-(5-Phenyl-1-(trimethylsilyl)pent-1-yn-3-yl)piperidin-4-one (2g)}

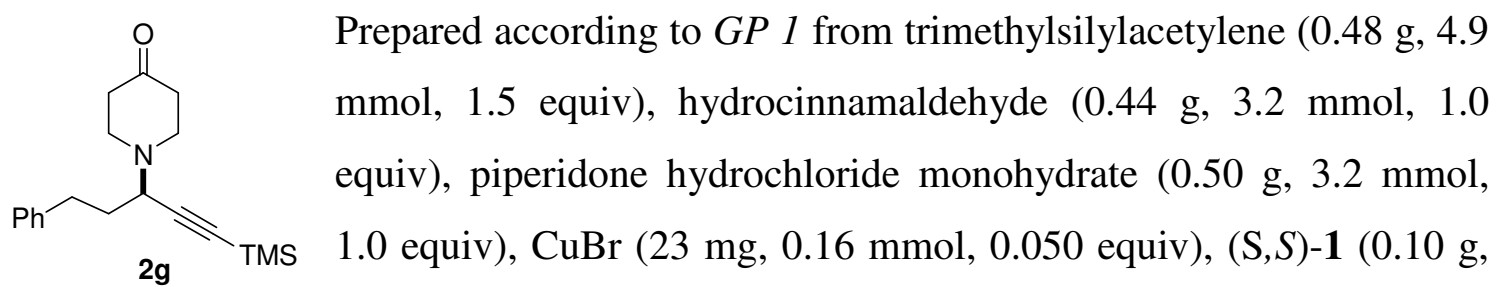

$0.18 \mathrm{mmol}, 0.055$ equiv), triethylamine ( $0.36 \mathrm{~g}, 3.6 \mathrm{mmol}, 1.1$ equiv) and $4 \AA$ molecular sieves $(1.6 \mathrm{~g})$ in $13 \mathrm{~mL}$ dichloromethane at $23{ }^{\circ} \mathrm{C}$ for 22 hours. Purification by flash column chromatography on silica gel afforded $0.86 \mathrm{~g}(84 \%)$ of the title compound as a yellowish soild: $\mathrm{mp} 52{ }^{\circ} \mathrm{C}$. The enantioselectivity was $85 \%$ ee as determined by HPLC (Daicel Chiralpak OD-H column 250 x $4.6 \mathrm{~mm}$ i.d and Daicel Chiralpak OD-H column $150 \times 4.6 \mathrm{~mm}$ i.d with hexane : 2-propanol $=98: 2$, flow rate $0.5 \mathrm{~mL} / \mathrm{min}$, UV fluorescence $\left.254 \mathrm{~nm} ; \mathrm{t}_{\mathrm{r}(\text { minor })}=34.0 \mathrm{~min}, \mathrm{t}_{\mathrm{r}(\text { major })}=42.0 \mathrm{~min}\right)$.

$[\alpha]_{D}^{25}=-4.3\left(c 0.8, \mathrm{CHCl}_{3}\right)$

${ }^{1} \mathbf{H}$ NMR $\left(300 \mathrm{MHz}, \mathrm{CDCl}_{3}\right) \delta$ 7.33-7.27 (m, $\left.2 \mathrm{H}\right), 7.24-7.18(\mathrm{~m}, 3 \mathrm{H}), 3.44(\mathrm{t}, J=7.8$ Hz, 1 H), 2.96-2.86 (m, 2 H), 2.83-2.76 (m, 2 H), 2.74-2.67 (m, 2 H), 2.48-2.29 (m, 4 H), 2.04-1.95 (m, $2 \mathrm{H}), 0.18$ (s, $9 \mathrm{H})$;

${ }^{13}$ C NMR $\left(75 \mathrm{MHz}, \mathrm{CDCl}_{3}\right) \delta 208.7,141.2,128.4,128.2,125.8,102.5,90.5,56.3,49.0$, $41.5,35.2,32.5,0.3$

FTIR (neat) v 3027, 2959, 2909, 2816, 2160, 1722, 1496, 1455, 1338, 1250, 1206, 1125 $\mathrm{cm}^{-1}$;

HRMS (EI) $m / z$ calculated for $\mathrm{C}_{19} \mathrm{H}_{27} \mathrm{NOSi}\left([\mathrm{M}]^{+}\right)$313.1857, found 313.1855. 


\section{(S)-1-(4-Methyl-1-phenylpent-1-yn-3-yl)piperidin-4-one (2h)}

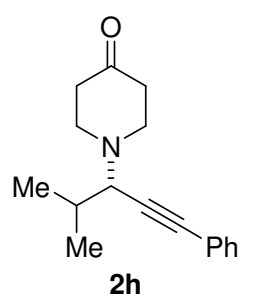

Prepared according to GP 2 from phenylacetylene $(153 \mathrm{mg}, 1.50 \mathrm{mmol}$, 1.00 equiv), 2-methylpropanal (216 mg, $3.00 \mathrm{mmol}, 2.00$ equiv), piperidone hydrochloride monohydrate $(230 \mathrm{mg}, 1.50 \mathrm{mmol}, 1.00$ equiv), $\mathrm{CuBr}$ (10.8 mg, $75.0 \mu \mathrm{mol}, 0.050$ equiv), $(R, R)-\mathbf{1}$. (46.2 mg, 82.5 $\mu \mathrm{mol}, 0.055$ equiv), triethylamine (167 mg, $1.65 \mathrm{mmol}, 1.10$ equiv) and $4 \AA$ molecular sieves $(0.750 \mathrm{~g})$ in dichloromethane $(6 \mathrm{~mL})$ at $23{ }^{\circ} \mathrm{C}$ for 22 hours. Purification by flash column chromatography on silica gel afforded $315 \mathrm{mg}(82 \%)$ of the title compound as a colorless soild: $\mathrm{mp} 70^{\circ} \mathrm{C}$.

The enantioselectivity was $85 \%$ ee as determined by HPLC (Daicel Chiralpak OD-H column 250 x $4.6 \mathrm{~mm}$ i.d and Daicel Chiralpak OD-H column 150 x $4.6 \mathrm{~mm}$ i.d with hexane : 2-propanol $=98.5: 1.5$, flow rate $0.25 \mathrm{ml} / \mathrm{min}$, $\mathrm{UV}$ fluorescence $254 \mathrm{~nm} ; \mathrm{t}_{\mathrm{r} \text { (major) }}$ $\left.=53.6 \mathrm{~min}, \mathrm{t}_{\mathrm{r}(\operatorname{minor})}=61.0 \mathrm{~min}\right)$.

$[\alpha]_{D}^{25}=-14.8\left(c 0.9, \mathrm{CHCl}_{3}\right)$

${ }^{1}$ H NMR (300 MHz, $\left.\mathrm{CDCl}_{3}\right) \delta$ 7.42-7.38 (m $\left.2 \mathrm{H}\right), 7.30-7.26(\mathrm{~m}, 3 \mathrm{H}), 3.19$ (d, $J=10.0$ Hz, 1 H), 3.03-2.94 (m, 2 H), 2.81-2.73 (m, 2 H), 2.57-2.41 (m, 4 H), 1.99-1.87 (m, 1 H), $1.11(\mathrm{dd}, J=20.2,6.5 \mathrm{~Hz}, 6 \mathrm{H})$;

${ }^{13}$ C NMR $\left(75 \mathrm{MHz}, \mathrm{CDCl}_{3}\right) \delta$ 209.2, 131.5, 128.8, 127.9, 123.0, 88.5, 86.2, 64.4, 49.5, 41.7, 31.2, 20.8, 20.0;

FTIR (thin film) v 3055, 2960, 2813, 2360, 1717, 1598, 1489, 1380, 1334, 1262, 1212, $1074 \mathrm{~cm}^{-1}$;

HRMS (EI) $m / z$ calculated for $\mathrm{C}_{17} \mathrm{H}_{20} \mathrm{NO}\left([\mathrm{M}-\mathrm{H}]^{+}\right)$254.1545, found 254.1543. 


\section{(R)-1-(4-Methyl-1-phenylpent-1-yn-3-yl)piperidin-4-one (ent-2h)}

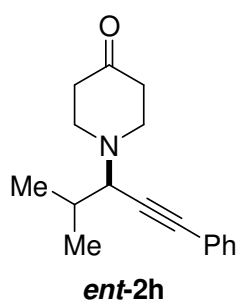

Prepared according to GP 2 from phenylacetylene $(51 \mathrm{mg}, 0.50 \mathrm{mmol}$, 1.0 equiv), 2-methylpropanal ( $72 \mathrm{mg}, 1.0 \mathrm{mmol}, 2.0$ equiv), piperidone hydrochloride monohydrate (77 mg, $0.50 \mathrm{mmol}, 1.0$ equiv), $\mathrm{CuBr}$ (3.6 $\mathrm{mg}, 0.025 \mathrm{mmol}, 0.050$ equiv), $(R, S)-1 .(15 \mathrm{mg}, 0.028 \mathrm{mmol}, 0.055$ equiv), triethylamine (56 mg, $0.55 \mathrm{mmol}, 1.1$ equiv) and $4 \AA$ molecular column chromatography on silica gel afforded $101 \mathrm{mg}(79 \%)$ of the title compound as a colorless soild: $\mathrm{mp} 70{ }^{\circ} \mathrm{C}$.

The enantioselectivity was $83 \%$ ee as determined by HPLC (Daicel Chiralpak OD-H column 250 x $4.6 \mathrm{~mm}$ i.d and Daicel Chiralpak OD-H column 150 x $4.6 \mathrm{~mm}$ i.d with hexane $:$-propanol $=98.5: 1.5$, flow rate $0.25 \mathrm{ml} / \mathrm{min}$, $\mathrm{UV}$ fluorescence $254 \mathrm{~nm} ; \mathrm{t}_{\mathrm{r}(\operatorname{minor})}$ $\left.=53.0 \mathrm{~min}, \mathrm{t}_{\mathrm{r}(\text { major })}=60.0 \mathrm{~min}\right)$.

$[\alpha]_{D}^{25}=+13.2\left(c 1.2, \mathrm{CHCl}_{3}\right)$

\section{(S)-1-(2-Methyl-7-phenylhept-4-yn-3-yl)piperidin-4-one (2i)}

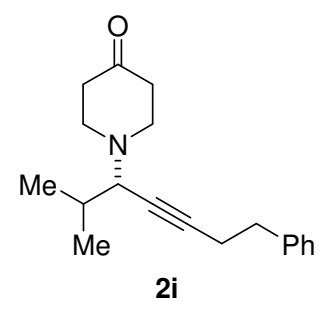

Prepared according to GP 2 from 4-phenyl-1-butyne (65 $\mathrm{mg}, 0.50$ mmol, 1.0 equiv), 2-methylpropanal (72 mg, $1.0 \mathrm{mmol}, 2.0$ equiv), piperidone hydrochloride monohydrate $(77 \mathrm{mg}, 0.50 \mathrm{mmol}, 1.0$ equiv), $\mathrm{CuBr}$ (3.6 mg, $0.025 \mathrm{mmol}, 0.050$ equiv), $(R, R)-\mathbf{1}$. (15 mg, $0.028 \mathrm{mmol}, 0.055$ equiv), triethylamine (56 $\mathrm{mg}, 0.55 \mathrm{mmol}, 1.1$ equiv) and $4 \AA$ molecular sieves $(0.25 \mathrm{~g})$ in $2 \mathrm{~mL}$ dichloromethane at $23{ }^{\circ} \mathrm{C}$ for 22 hours. Purification by flash column chromatography on silica gel afforded $102 \mathrm{mg}$ (72\%) of the title compound as a colorless oil. The enantioselectivity was $70 \%$ ee as determined by HPLC (Daicel Chiralpak OD-H column 250 x $4.6 \mathrm{~mm}$ i.d and Daicel Chiralpak OD-H column 150 x $4.6 \mathrm{~mm}$ i.d with hexane : 2-propanol $=99.5: 0.5$, flow rate $0.25 \mathrm{ml} / \mathrm{min}$, UV fluorescence $\left.254 \mathrm{~nm} ; \mathrm{t}_{\mathrm{r}(\text { major })}=51.8 \mathrm{~min}, \mathrm{t}_{\mathrm{r}(\text { major })}=57.0 \mathrm{~min}\right)$. 
$[\alpha]_{D}^{34}=-15.9\left(c 1.4, \mathrm{CHCl}_{3}\right)$

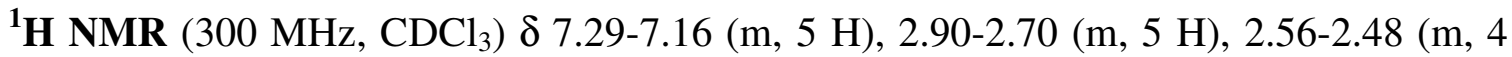
H), 2.45-2.31 (m, $4 \mathrm{H}), 1.73(\mathrm{~m}, 5 \mathrm{H}), 0.84(\mathrm{~m}, 1 \mathrm{H}), 0.99(\mathrm{dd}, J=8.8,6.6 \mathrm{~Hz}, 6 \mathrm{H})$;

${ }^{13}$ C NMR $\left(75 \mathrm{MHz}, \mathrm{CDCl}_{3}\right) \delta 209.3,140.5,128.4,128.2,126.2,85.4,77.2,64.0,49.2$, 41.6, 35.3, 31.1, 20.6, 19.9;

FTIR (thin film) v 3027, 2957, 2814, 1718, 1603, 1496, 1454, 1380, 1335, 1262, 1213, $1131,1114,1075 \mathrm{~cm}^{-1}$;

HRMS (EI) $m / z$ calculated for $\mathrm{C}_{19} \mathrm{H}_{25} \mathrm{NO}\left([\mathrm{M}]^{+}\right)$283.1936, found 238.1936.

\section{(S)-4-Methyl-1-phenylpent-1-yn-3-amine (4)}

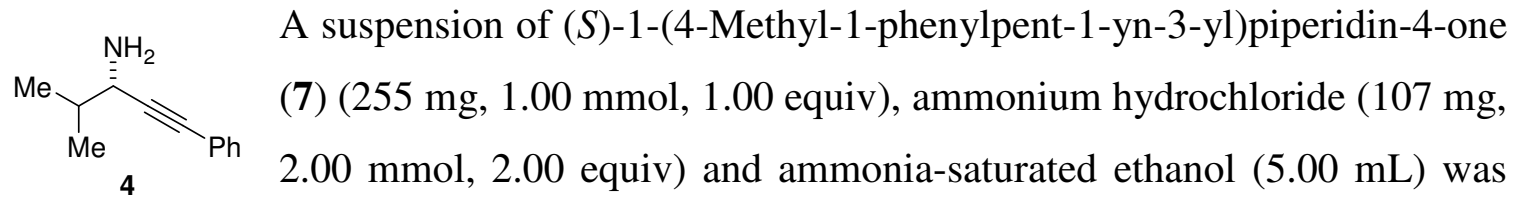
stirred in a pressure tube at $90{ }^{\circ} \mathrm{C}$ for 14 hours. Removal of the solvent under reduced pressure afforded a dark yellow oil which was purified by flash chromatography on silica gel using 5\% MeOH in EtOAc as eluent affording $129 \mathrm{mg}(75 \%)$ of the title compound as a yellowish oil.

$[\alpha]_{D}^{26}=-2.8\left(c 0.8, \mathrm{CHCl}_{3}\right)$

${ }^{1} \mathbf{H}$ NMR $\left(300 \mathrm{MHz}, \mathrm{CDCl}_{3}\right) \delta$ 7.44-7.38 (m, $\left.2 \mathrm{H}\right), 7.33-7.28(\mathrm{~m}, 3 \mathrm{H}), 3.62(\mathrm{~d}, J=5.4$ Hz, $1 \mathrm{H}), 1.94-1.79$ (m, $1 \mathrm{H}), 1.54$ (s, $2 \mathrm{H}), 1.06$ (dd, $J=6.7,1.3 \mathrm{~Hz}, 6 \mathrm{H})$;

${ }^{13}$ C NMR $\left(75 \mathrm{MHz}, \mathrm{CDCl}_{3}\right) \delta 131.4,128.1,127.7,123.3,91.6,83.2,50.2,35.0,19.4$, 17.8 ; 
FTIR (thin film) v 3373, 3056, 2959, 2870, 1598, 1489, 1466, 1442, 1383, 1366, 1277, $1070 \mathrm{~cm}^{-1}$;

HRMS (EI) $\mathrm{m} / z$ calculated for $\mathrm{C}_{12} \mathrm{H}_{15} \mathrm{~N}\left([\mathrm{M}]^{+}\right)$173.1204, found 173.1202 .

\section{Deprotections using aminomethylated polystyrene resin EHL}

\section{General Procedure 2 (GP 2)}

A pressure tube equipped with a magnetic stirring bar was charged with the desired piperidone $(1 \mathrm{mmol})$, aminomethylated polystyrene EHL $(0.50 \mathrm{~g}, 1.5 \mathrm{mmol}, 3.0 \mathrm{mmol} / \mathrm{g}$ loading) and $\mathrm{NH}_{4} \mathrm{Cl}(1 \mathrm{mmol})$. The mixture was suspended in anydrous EtOH $(5.0 \mathrm{~mL})$ and the tube was sealed and immersed in an oil bath, preheated to $100{ }^{\circ} \mathrm{C}$. Upon consumption of the piperidone (as judged by TLC analysis), the reaction was cooled to r.t. and filtered. The filter cake was washed with $\mathrm{MeOH}$ and filtered. The mixture was treated with $\mathrm{K}_{2} \mathrm{CO}_{3}(1 \mathrm{mmol})$ and stirred for 2-5 hours followed by aqueous work-up. The organic layers were cooled to $0{ }^{\circ} \mathrm{C}$ and treated with a freshly prepared solution of $\mathrm{HCl}$ (2.0 mmol, 1.0 M in $\mathrm{MeOH}$ ) and concentrated. The residue was dissolved in $\mathrm{MeOH}$, treated with activated charcoal, filtered and concentrated to afford the desired propargyl amine hydrochloride salt.

\section{(S)-4-Methyl-1-phenylpent-1-yn-3-amine hydrochloride (5h)}

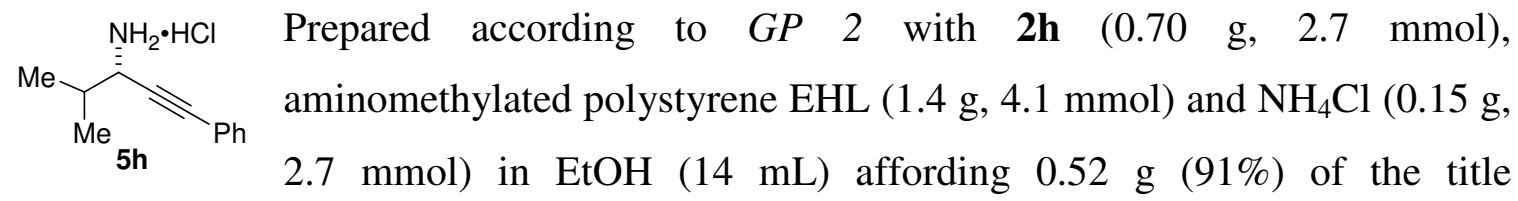
compound as a light yellow solid.

$[\alpha]_{D}^{28}=-3.1\left(c 1.4, \mathrm{CH}_{3} \mathrm{OH}\right)$

${ }^{1} \mathbf{H}$ NMR $\left(300 \mathrm{MHz}, \mathrm{CD}_{3} \mathrm{OD}\right) \delta$ 7.50-7.46 (m, $\left.2 \mathrm{H}\right), 7.41-7.35(\mathrm{~m}, 3 \mathrm{H}), 4.25(\mathrm{~d}, J=5.4$ $\mathrm{Hz}, 1 \mathrm{H}), 2.26-2.16(\mathrm{~m}, 1 \mathrm{H}), 1.17(\mathrm{~d}, J=6.3 \mathrm{~Hz}, 3 \mathrm{H}), 1.15(\mathrm{~d}, J=6.6 \mathrm{~Hz}, 3 \mathrm{H})$; 
${ }^{13}$ C NMR (75 MHz, CD 3 OD) $\delta 132.4,130.0,129.3,122.3,88.74,82.5,50.1,32.4,19.3$, 16.9 ;

FTIR (neat) $v$ 2923, 2856, 2047, 1595, 1514, 1366, $1293 \mathrm{~cm}^{-1}$;

HRMS (MALDI) $m / z$ calculated for $\mathrm{C}_{12} \mathrm{H}_{16} \mathrm{~N}\left([\mathrm{M}+1]^{+}\right)$174.1277, found 174.1277.

\section{(S)-Oct-1-yn-3-amine (5a)}

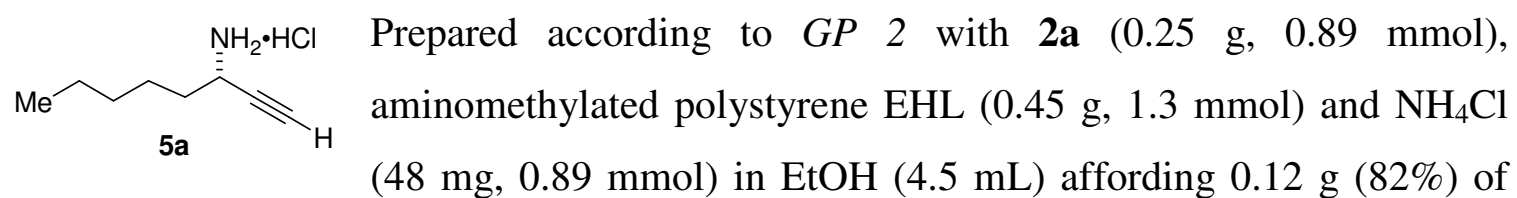
the title compound as a yellow solid containing an unidentified impurity. Purification of the free amine by chromatography on $\mathrm{SiO}_{2}(95: 5, \mathrm{EtOAc} / \mathrm{MeOH})$ followed by treatment with $\mathrm{HCl}(1.0 \mathrm{M}$ in $\mathrm{MeOH})$ afforded a colorless solid.

$[\alpha]_{D}^{28}=-7.3\left(c 0.2, \mathrm{CH}_{3} \mathrm{OH}\right)$

${ }^{1} \mathbf{H}$ NMR $\left(300 \mathrm{MHz}, \mathrm{CD}_{3} \mathrm{OD}\right) \delta 4.08(\mathrm{ddd}, J=8.1,5.4,2.1 \mathrm{~Hz}, 1 \mathrm{H}), 3.22(\mathrm{~d}, J=2.1 \mathrm{~Hz}$, $1 \mathrm{H}), 1.90-1.71(\mathrm{~m}, 2 \mathrm{H}), 1.62-1.48(\mathrm{~m}, 2 \mathrm{H}), 1.43-1.35$ (m, $4 \mathrm{H}), 0.94(\mathrm{t}, J=6.6 \mathrm{~Hz}, 3$ $\mathrm{H})$;

${ }^{13}$ C NMR (75 MHz, $\left.\mathrm{CD}_{3} \mathrm{OD}\right) \delta 79.4,77.9,44.2,34.6,32.2,26.2,23.5,14.4$

FTIR (neat) v 3263, 2931, 2122, 1598, 1508, 1466, 1409, 1380, 1134, 1088, $1048 \mathrm{~cm}^{-1}$; 


\section{(S)-4-Methylpent-1-yn-3-amine hydrochloride (5b)}

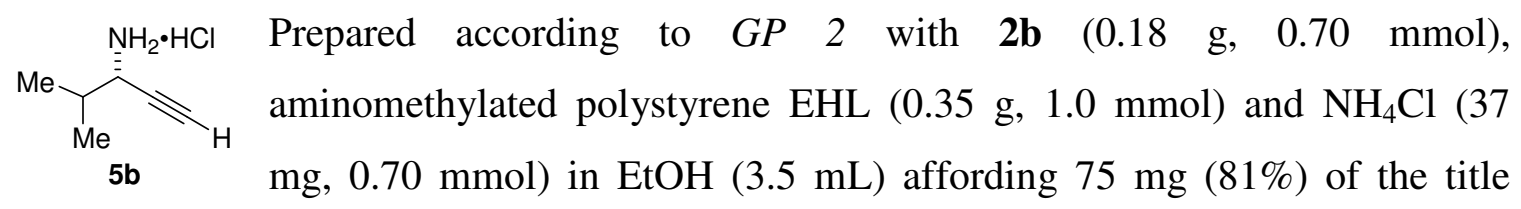
compound as a light yellow solid.

$[\alpha]_{D}^{28}=-11.9\left(c 0.4, \mathrm{CH}_{3} \mathrm{OH}\right)$

${ }^{1}$ H NMR $\left(300 \mathrm{MHz}, \mathrm{CD}_{3} \mathrm{OD}\right) \delta 4.01(\mathrm{dd}, J=5.4,2.4 \mathrm{~Hz}, 1 \mathrm{H}), 3.22(\mathrm{~d}, J=2.4 \mathrm{~Hz}, 1 \mathrm{H})$, 2.14-2.04 (m, $1 \mathrm{H}), 1.10(\mathrm{~d}, J=6.6 \mathrm{~Hz}, 3 \mathrm{H}), 1.08(\mathrm{~d}, J=6.9 \mathrm{~Hz}, 3 \mathrm{H})$;

${ }^{13}$ C NMR (75 MHz, CD 3 OD) $\delta$ 78.4, 77.3, 49.4 (obscured by solvent), 32.1, 19.1, 16.8;

FTIR (neat) v 3251, 2968, 2926, 2123, 1467, 1375, $1161 \mathrm{~cm}^{-1}$;

\section{(S)-4-Ethylhex-1-yn-3-amine hydrochloride (5c)}

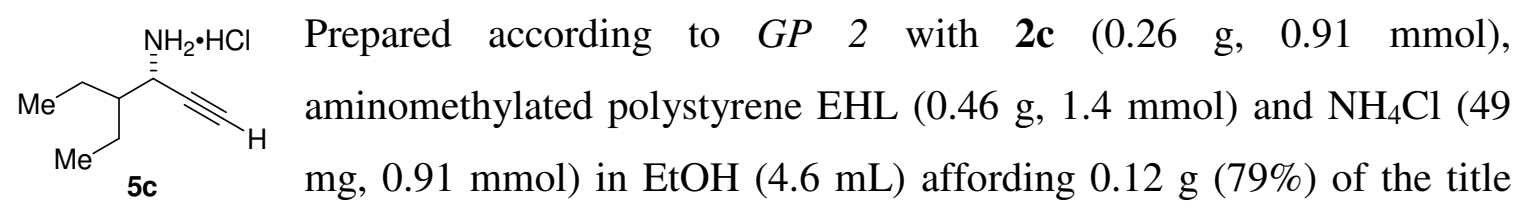
compound as a yellowish foam.

$[\alpha]_{D}^{23}=+3.8\left(c 0.5, \mathrm{CHCl}_{3}\right)$

${ }^{1}$ H NMR (300 MHz, CD 3 OD) $\delta 4.17(\mathrm{dd}, J=3.9,2.4 \mathrm{~Hz}, 1 \mathrm{H}), 3.20(\mathrm{~d}, \mathrm{~J}=2.4 \mathrm{~Hz}, 1 \mathrm{H})$, 1.68-1.54 (m, $3 \mathrm{H}), 1.52-1.42(\mathrm{~m}, 2 \mathrm{H}), 0.98$ (t, $J=7.2 \mathrm{~Hz}, 3 \mathrm{H}), 0.98$ (t, $J=7.5 \mathrm{~Hz}, 3 \mathrm{H})$;

${ }^{13}$ C NMR (75 MHz, $\left.\mathrm{CD}_{3} \mathrm{OD}\right) \delta 78.4,77.5,46.4,44.6,23.0,22.0,11.1,11.0 ;$

FTIR (neat) v 3255, 2963, 2932, 2878, 2121, 1518, 1461, 1410, 1381, $1164 \mathrm{~cm}^{-1}$; 
(R)-1-Cyclohexylprop-2-yn-1-amine hydrochloride (5e)

$\underbrace{\mathrm{H}_{\mathrm{H}} \cdot \mathrm{HCl}}_{5 \mathbf{e}} \begin{aligned} & \text { Prepared according to } G P \\ & \text { aminomethylated polystyrene EHL }(0.33 \mathrm{~g}, 0.98 \mathrm{mmol}) \text { and } \mathrm{NH}_{4} \mathrm{Cl}(35\end{aligned}$ compound as a light yellow solid.

$[\alpha]_{D}^{27}=-10.2\left(c 1.4, \mathrm{CH}_{3} \mathrm{OH}\right)$

${ }^{1}$ H NMR $\left(300 \mathrm{MHz}, \mathrm{CD}_{3} \mathrm{OD}\right) \delta 3.96(\mathrm{dd}, J=5.4,2.1 \mathrm{~Hz}, 1 \mathrm{H}), 3.22(\mathrm{~d}, J=2.4 \mathrm{~Hz}, 1 \mathrm{H})$, 1.91-1.70 (m, $6 \mathrm{H}), 1.40-1.17$ (m, $5 \mathrm{H})$;

${ }^{13}$ C NMR (75 MHz, CD $\left.3 \mathrm{OD}\right) \delta 78.5,77.8,41.4,30.1,28.1,26.7,26.5,26.3$;

FTIR (neat) v 3246, 2923, 2852, 2123, 1589, 1515, 1478, 1354, $1179 \mathrm{~cm}^{-1}$;

HRMS (EI) $m / z$ calculated for $\mathrm{C}_{9} \mathrm{H}_{16} \mathrm{~N}\left([\mathrm{M}+1]^{+}\right)$138.1277, found 138.1277.

\section{(R)-1-Cyclopropylprop-2-yn-1-amine hydrochloride (5f)}

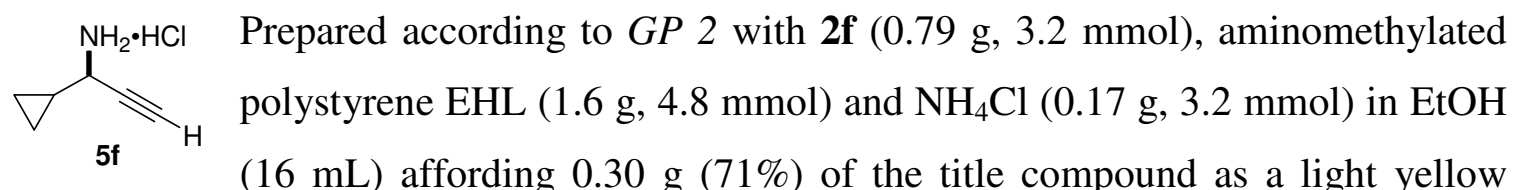
foam.

$[\alpha]_{D}^{26}=-38.2\left(c 0.9, \mathrm{CH}_{3} \mathrm{OH}\right)$

${ }^{1}$ H NMR (300 MHz, CD $\left.{ }_{3} \mathrm{OD}\right) \delta 3.93(\mathrm{dd}, J=7.2,2.1 \mathrm{~Hz}, 1 \mathrm{H}), 3.20(\mathrm{~d}, J=2.1 \mathrm{~Hz}, 1 \mathrm{H})$, 1.33-1.25 (m, $1 \mathrm{H}), 0.74-0.68$ (m, $2 \mathrm{H}), 0.61-0.56$ (m, $2 \mathrm{H})$; 
${ }^{13}$ C NMR (75 MHz, $\left.\mathrm{CD}_{3} \mathrm{OD}\right) \delta$ 77.5. 77.1, 47.3, 13.6, 4.1, 2.5;

FTIR (neat) $\vee 3244,2978,2924,2864,2125,1595,1510,1360,1031 \mathrm{~cm}^{-1}$;

\section{(R)-5-Phenylpent-1-yn-3-amine hydrochloride (5g)}

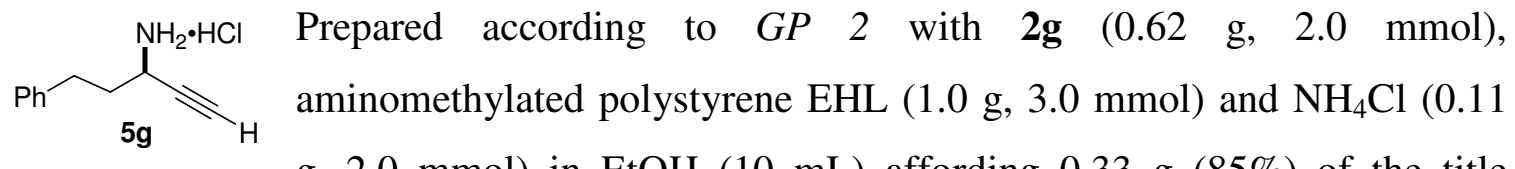
$\mathrm{g}, 2.0 \mathrm{mmol})$ in EtOH $(10 \mathrm{~mL})$ affording $0.33 \mathrm{~g}(85 \%)$ of the title compound as a light yellow solid.

$[\alpha]_{D}^{24}=-33.6\left(c\right.$ 1.2, $\left.\mathrm{CHCl}_{3}\right) ;$

${ }^{1} \mathrm{H}$ NMR (300 MHz, CD $\left.\mathrm{OD}\right) \delta$ 7.33-7.17 (m, $\left.5 \mathrm{H}\right), 4.03(\mathrm{ddd}, J=9.6,4.8,2.1 \mathrm{~Hz}, 1 \mathrm{H})$, $3.32(\mathrm{~d}, J=2.1 \mathrm{~Hz}, 1 \mathrm{H}), 2.96-2.73(\mathrm{~m}, 2 \mathrm{H}), 2.23-2.01(\mathrm{~m}, 2 \mathrm{H})$;

${ }^{13}$ C NMR (75 MHz, $\left.\mathrm{CD}_{3} \mathrm{OD}\right) \delta$ 140.7, 129.3, 129.1, 127.1, 78.8, 78.4, 43.5, 36.0, 32.3;

FTIR (neat) v 3269, 2928, 2793, 2230, 2119, 1497, 1457, 1356, $1161 \mathrm{~cm}^{-1}$; 
LOC ETHZ NMR Mercury-vx 300MHz Nr.6 04/13/05 10:12:16 USER:easchw GROUP:carrei SAMPLE:PA_1108h

STANDARD 1 H OBSERVE

File: PROTON

Pulse Sequence: s2pul

Solvent: $\mathrm{CDCl} 3$

Ambient temperature

Mercury-300BB "gem6oc"

Pulse 30.0 degrees

Acq. time $3.138 \mathrm{sec}$

Width $5099.4 \mathrm{~Hz}$

16 repetitions

OBSERVE H1, 300.2230602 MHz

DATA PROCESSING

FT size 32768

Total time $0 \mathrm{~min}, 52 \mathrm{sec}$
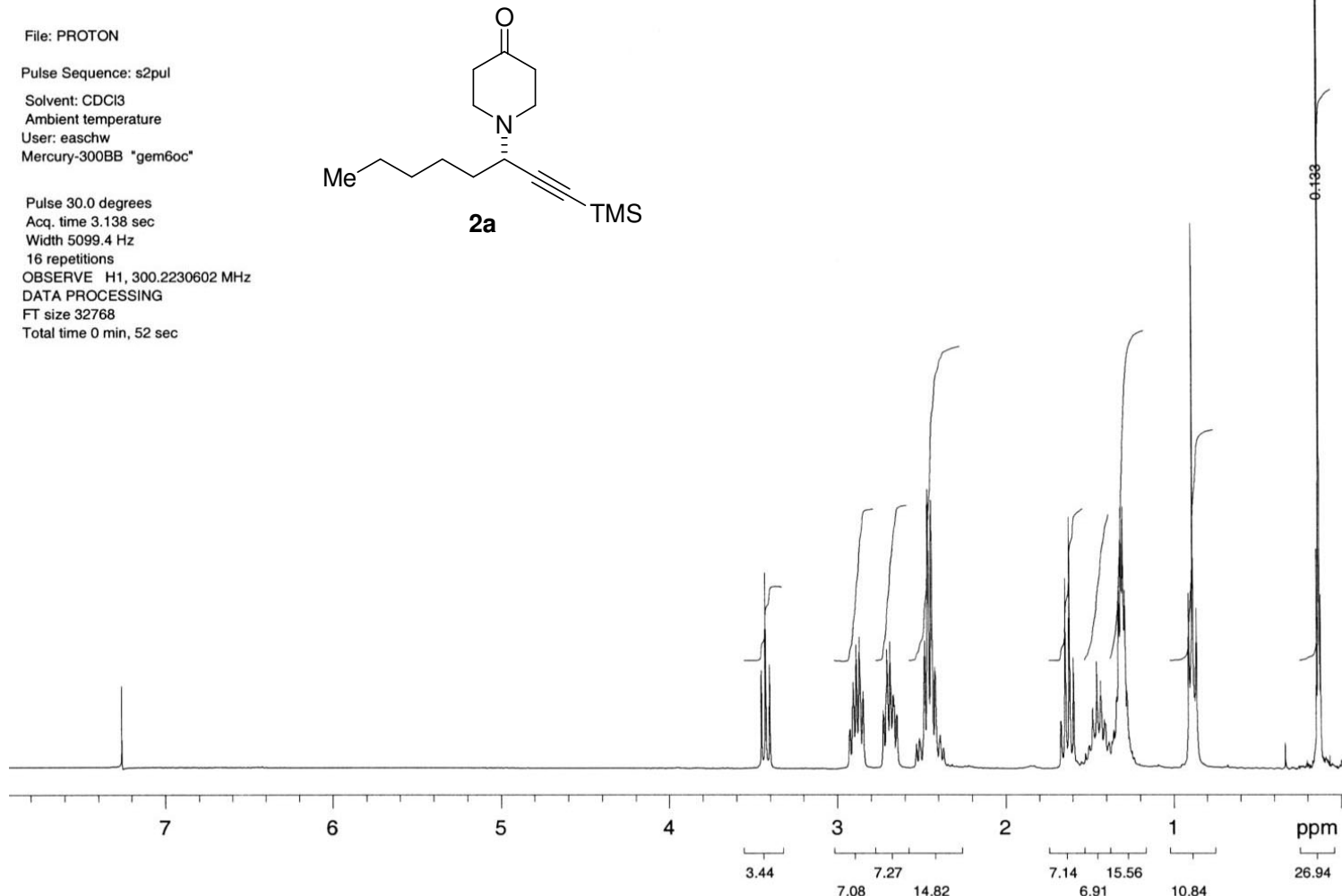

LOC ETHZ NMR Mercury-vx 300MHz Nr.6 04/13/05 10:25:51 USER:easchw GROUP:carrei SAMPLE:PA_1108C

13C OBSERVE

File: CARBON

Pulse Sequence: s2pul

Solvent: $\mathrm{CDCl} 3$

Ambient temperature

User: easchw

Mercury-300BB "gem6oc"

Relax. delay $1.000 \mathrm{sec}$

Pulse 45.0 degrees

Acq. time $1.300 \mathrm{sec}$

Width $20000.0 \mathrm{~Hz}$

300 repetitions

OBSERVE C13, $75.4911544 \mathrm{MHz}$

DECOUPLE H1, 300.2242455 MHz

Power $35 \mathrm{~dB}$

continuously on

WALTZ-16 modulated

Line broadening $20 \mathrm{~Hz}$

Line broadening $2.0 \mathrm{~Hz}$

Total time $12 \mathrm{~min}, 41 \mathrm{sec}$

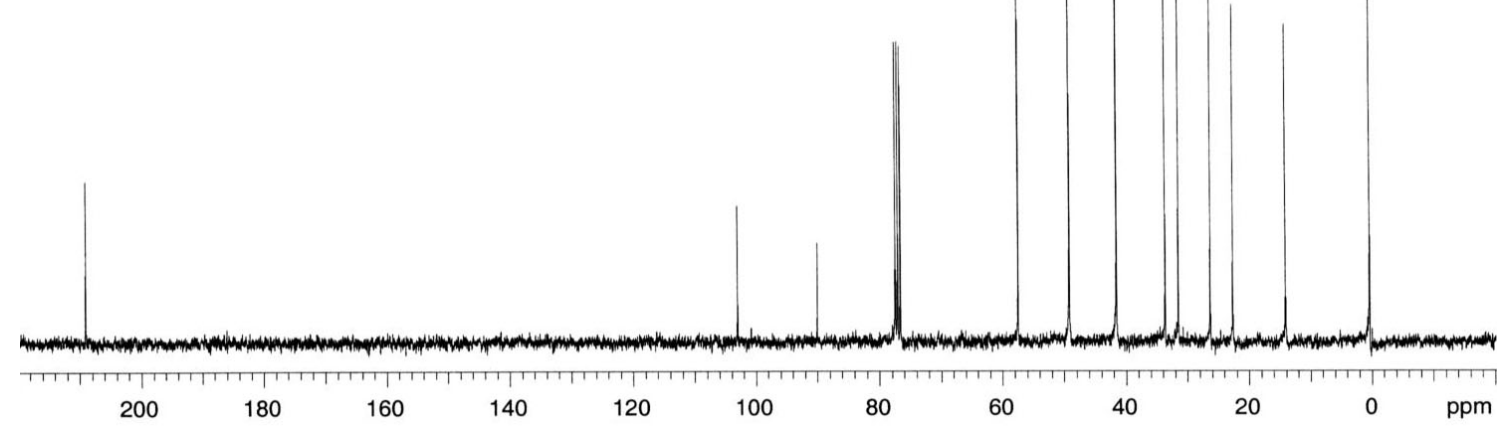


LOC ETHZ NMR Mercury-vx 300MHz Nr.6 04/15/05 09:55:49 USER:easchw GROUP:carrei SAMPLE:PA_1102h STANDARD 1 H OBSERVE

Pulse Sequence: s2pul Solvent: $\mathrm{CDCl} 3$ Ambient temperature
User: easchw

File: PA $1102 \mathrm{~h}$

UNITYplus-300 "nmroc"

Pulse 30.0 degrees

Acq. time $3.138 \mathrm{sec}$

Width $5099.4 \mathrm{~Hz}$

16 repettitions

$230602 \mathrm{MH}$

DATA PROCESSING

Total time $0 \mathrm{~min}, 50 \mathrm{sec}$

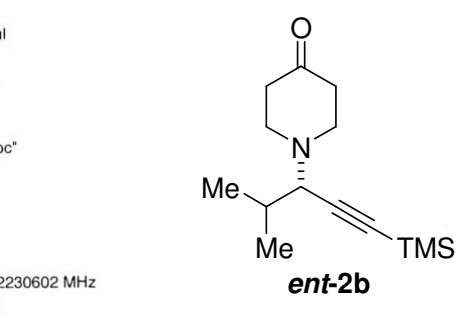

(1)

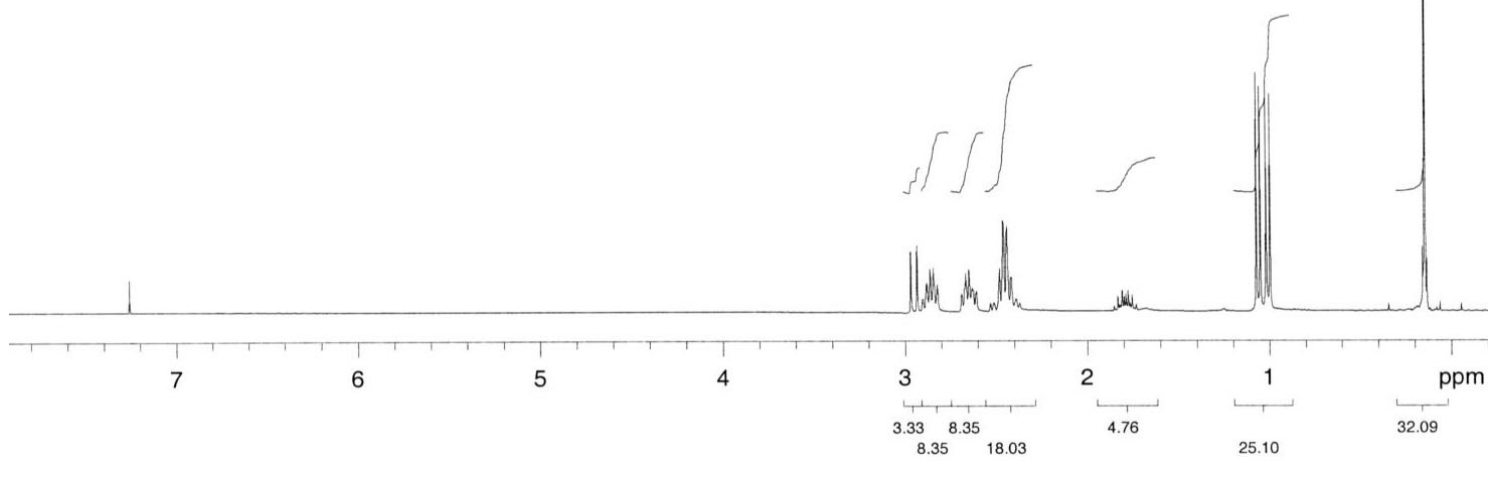

LOC ETHZ NMR Mercury-vx 300MHz Nr.6 04/15/05 10:02:12 USER:easchw GROUP:carrei SAMPLE:PA_1102C

13C OBSERVE

File: CARBON

Pulse Sequence: s2pul

Solvent: $\mathrm{CDCl} 3$

Ambient temperature

User. easchw

Mercury-300BB "gem6oc

Relax. delay $1.000 \mathrm{sec}$

Pulse 45.0 degrees

Width $20000.0 \mathrm{~Hz}$

OBSERVE C13, $75.4911544 \mathrm{MHz}$

DECOUPLE H1, 300.2242455 MHz

Power $35 \mathrm{~dB}$

continuously on

WALTZ-16 modulated

DATA PROCESSING

Line broadening $2.0 \mathrm{~Hz}$

Total time $16 \mathrm{~min}, 54 \mathrm{sec}$

25.10

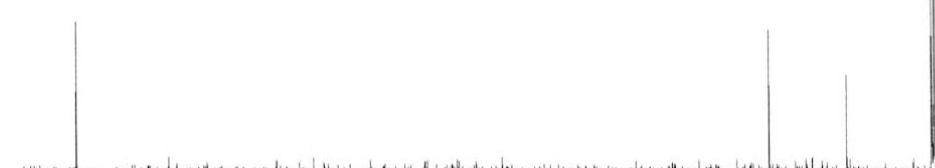

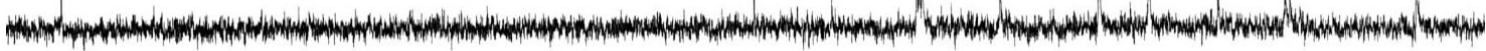

$\begin{array}{llllllllllllll} & 200 & 180 & 160 & 140 & 120 & 100 & 80 & 60 & 40 & 20 & \mathrm{ppm}\end{array}$


LOC ETHZ NMR Mercury-vx 300MHz Nr.6 04/18/05 15:37:59 USER:easchw GROUP:carrei SAMPLE:PA_1112h STANDARD 1 H OBSERVE

Pulse Sequence: s2pul

Solvent: $\mathrm{CDCl} 3$

Ambient temperature

User: easchw

UNITYplus-300 "nmroc"

Pulse 30.0 degrees

Acq. time $3.138 \mathrm{sec}$

16 repetitions

16 repetitions

DATA PROCESSING

FT size 32768

Total time $0 \mathrm{~min}, 50 \mathrm{sec}$
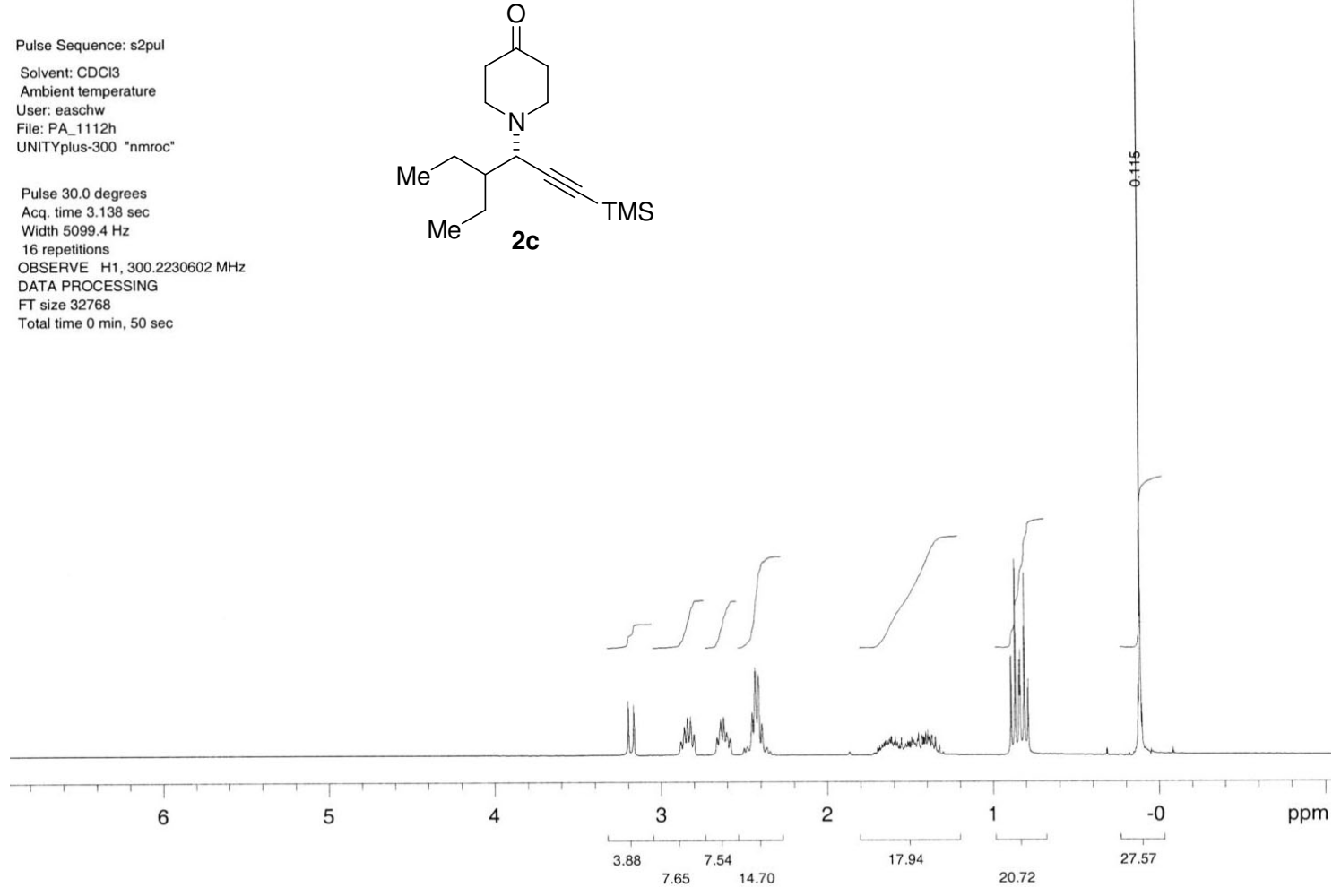

LOC ETHZ NMR Mercury-vx 300MHz Nr.6 04/18/05 15:40:51 USER:easchw GROUP:carrei SAMPLE:PA_1112C

13C OBSERVE

File: CARBON

Pulse Sequence: s2pul

Solvent: $\mathrm{CDCl} 3$

Ambient temperature

User: easchw

Mercury-300BB "gem6oc"

Relax. delay $1.000 \mathrm{se}$

Pulse 45.0 degrees

Acq. time $1.300 \mathrm{sec}$

Width $20000.0 \mathrm{~Hz}$

OBSERVE C13, $75.4911544 \mathrm{MHz}$

FCOUPLE H1, $300.2242455 \mathrm{MH}$

(1)

continuously on

WALTZ-16 modulate

DATA PROCESSING

Line broadening $2.0 \mathrm{~Hz}$

FT size 65536

Total time $12 \mathrm{~min}, 41 \mathrm{sec}$

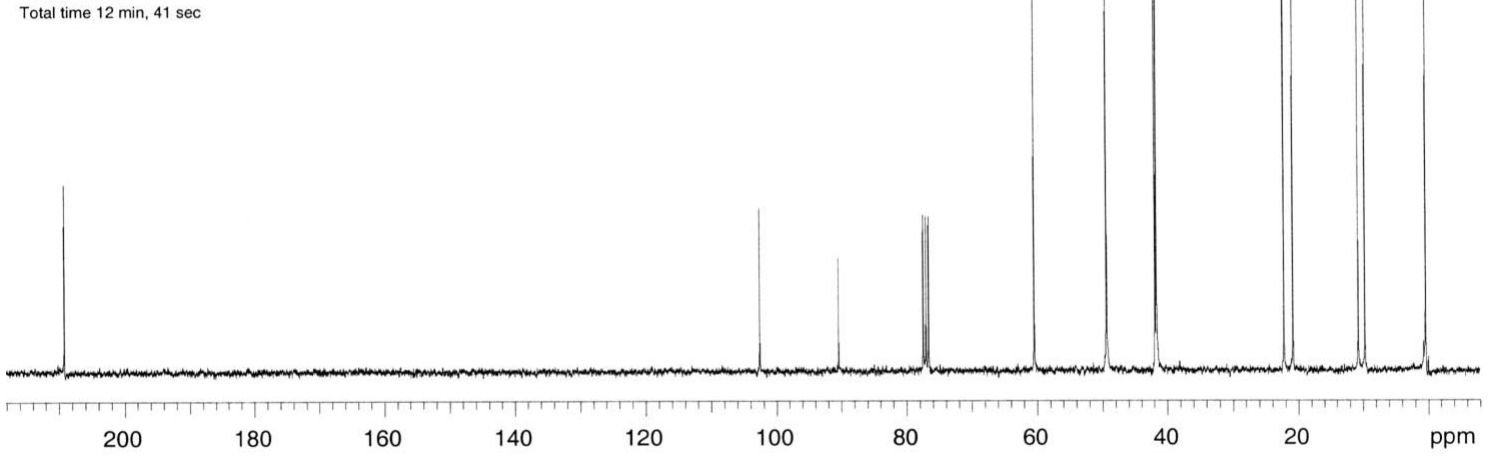


LOC ETHZ NMR Mercury-vx 300MHz Nr.6 04/14/05 14:46:24 USER:easchw GROUP:carrei SAMPLE:PA_1106h STANDARD 1 H OBSERVE

File: PROTON

Pulse Sequence: s2pul

Solvent: $\mathrm{CDCl} 3$

Ambient temperature

Mercury-300BB "gem6oc"

Pulse 30.0 degrees

Acq. time $3.138 \mathrm{sec}$

Width $5099.4 \mathrm{~Hz}$

16 repetitions

DATA PROCESSING

FT size 32768

Total time $0 \mathrm{~min}, 52 \mathrm{sec}$
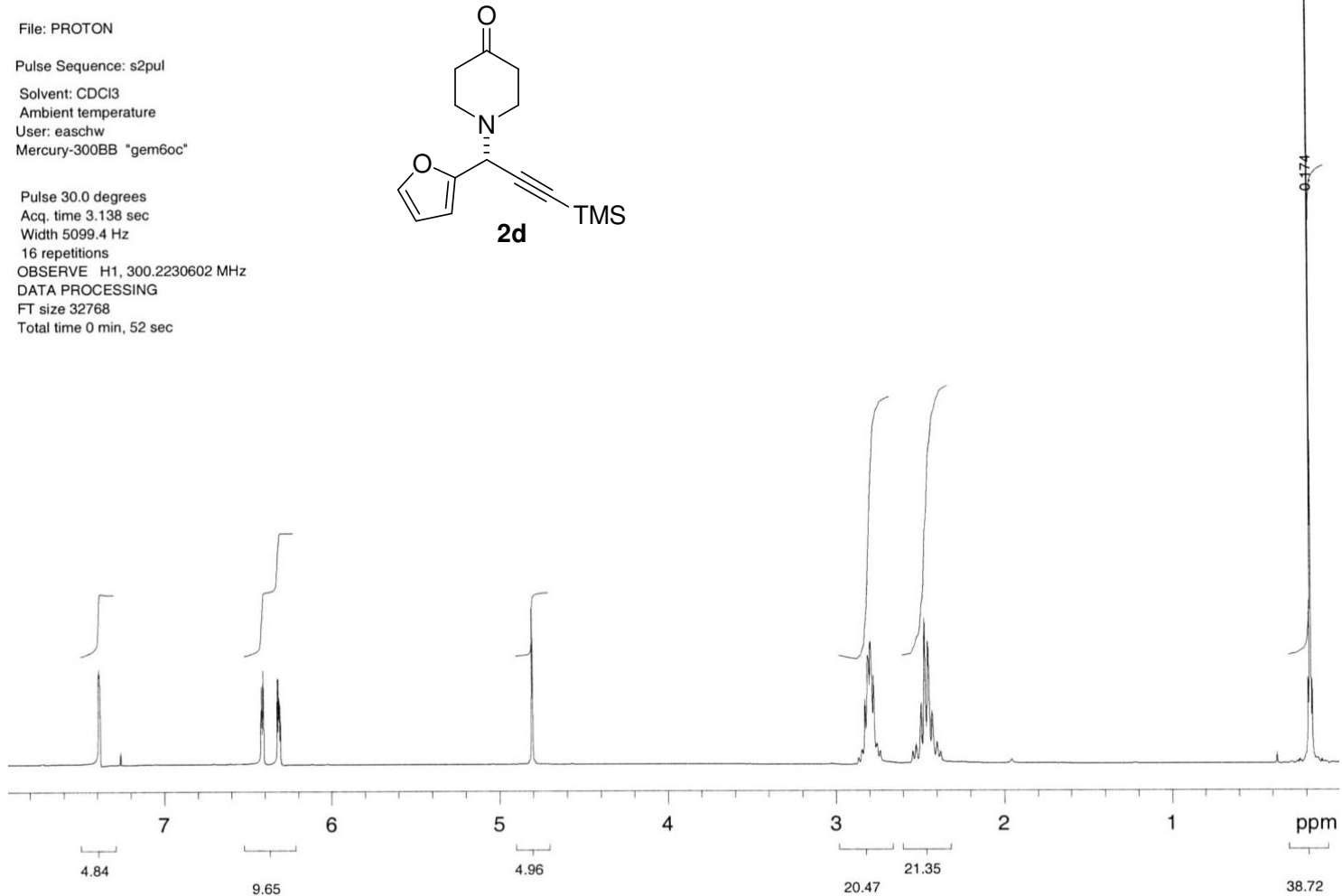

LOC ETHZ NMR Mercury-vx 300MHz Nr.6 04/14/05 15:03:06 USER:easchw GROUP:carrei SAMPLE:PA_1106c

13C OBSERVE

Pulse Sequence: s2pul

Solvent: CDC13

Ambient temperatur

User: easchw

File: PA_1106C
Mercury-300BB "gem6oc"

Relax. delay $1.000 \mathrm{sec}$

Pulse 45.0 degrees

Acq. time $1.300 \mathrm{sec}$

Width $20000.0 \mathrm{~Hz}$

300 repetitions

OBSERVE C13, $75.4911544 \mathrm{MHz}$

DECOUPLE H1, $300.2242455 \mathrm{MHz}$

Power $35 \mathrm{~dB}$

Continuously on
WALTZ-16 modulated

DATA PROCESSING

DATA PROCESSNG

FT size 65536

Total time $12 \mathrm{~min}, 41 \mathrm{sec}$

20.47

38.72

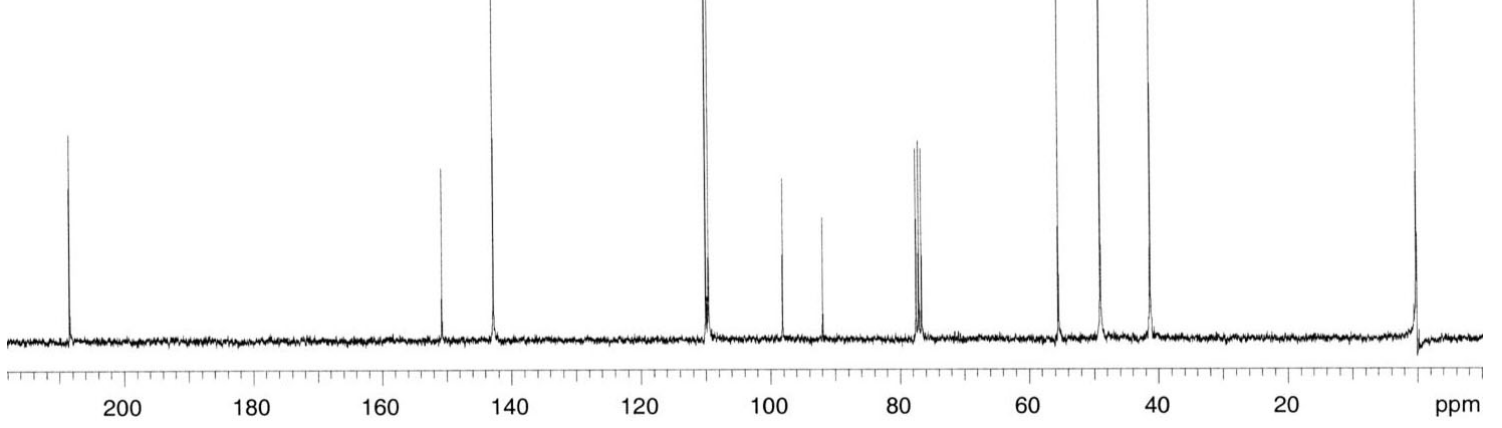



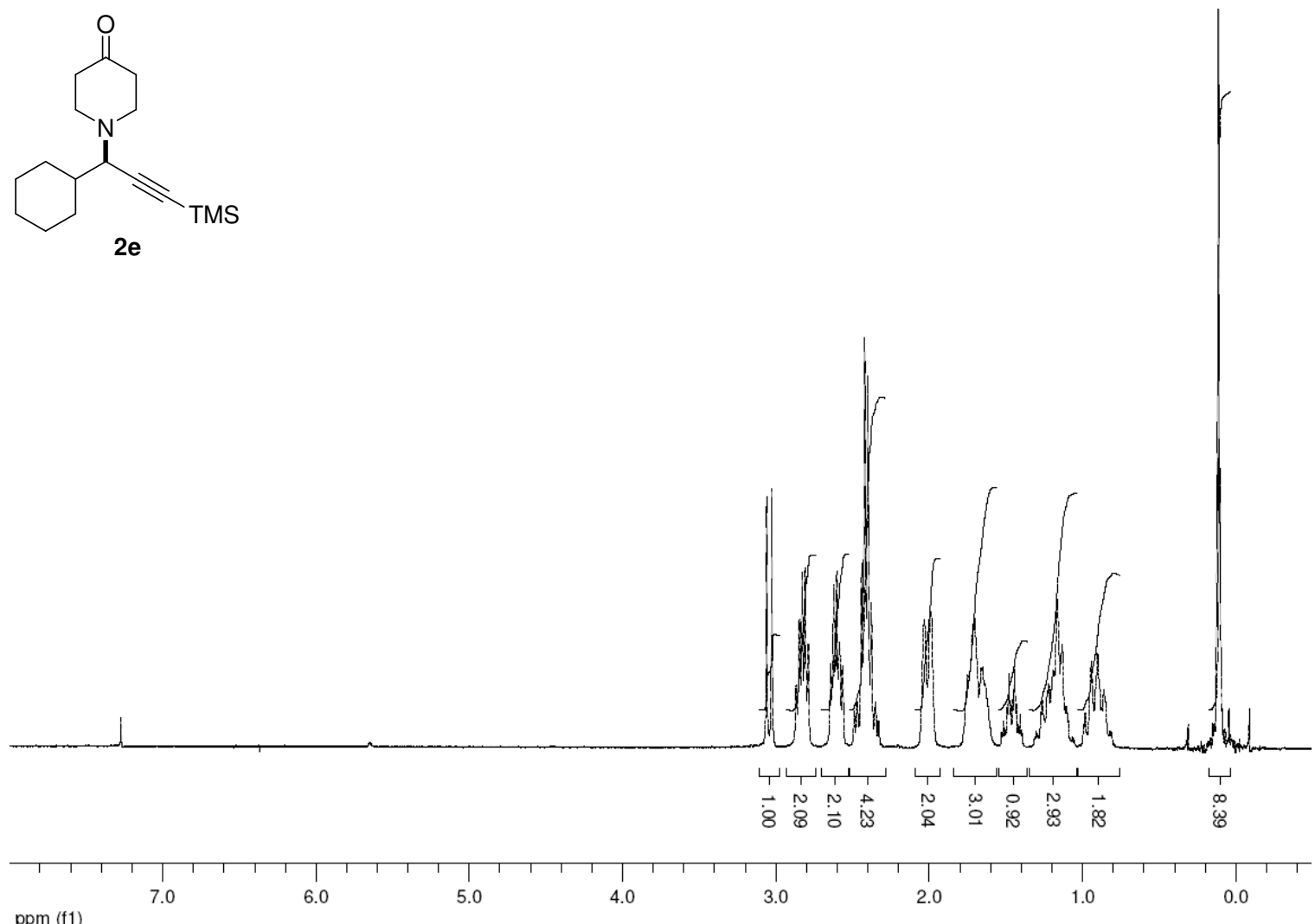

ppm (f1)
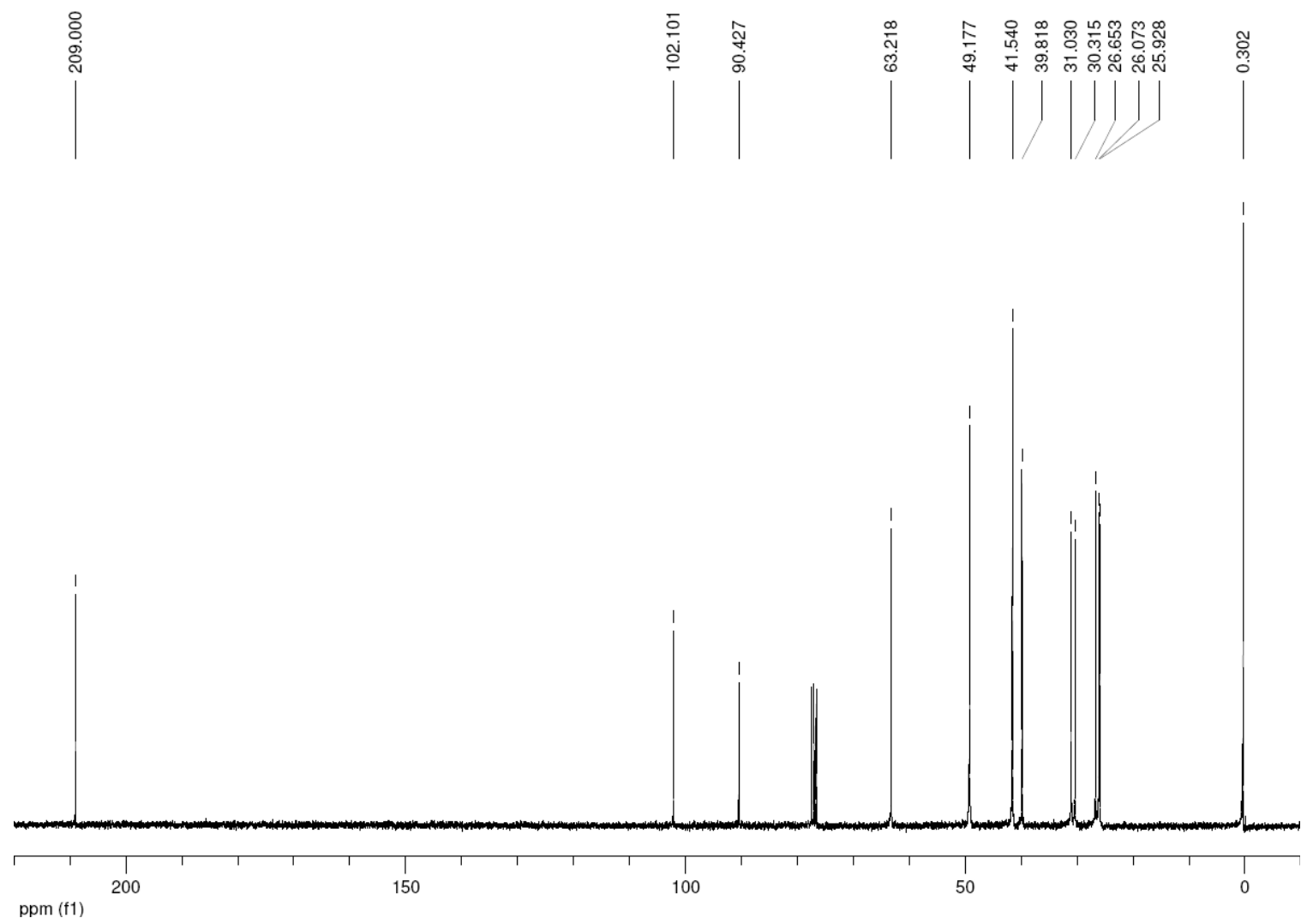

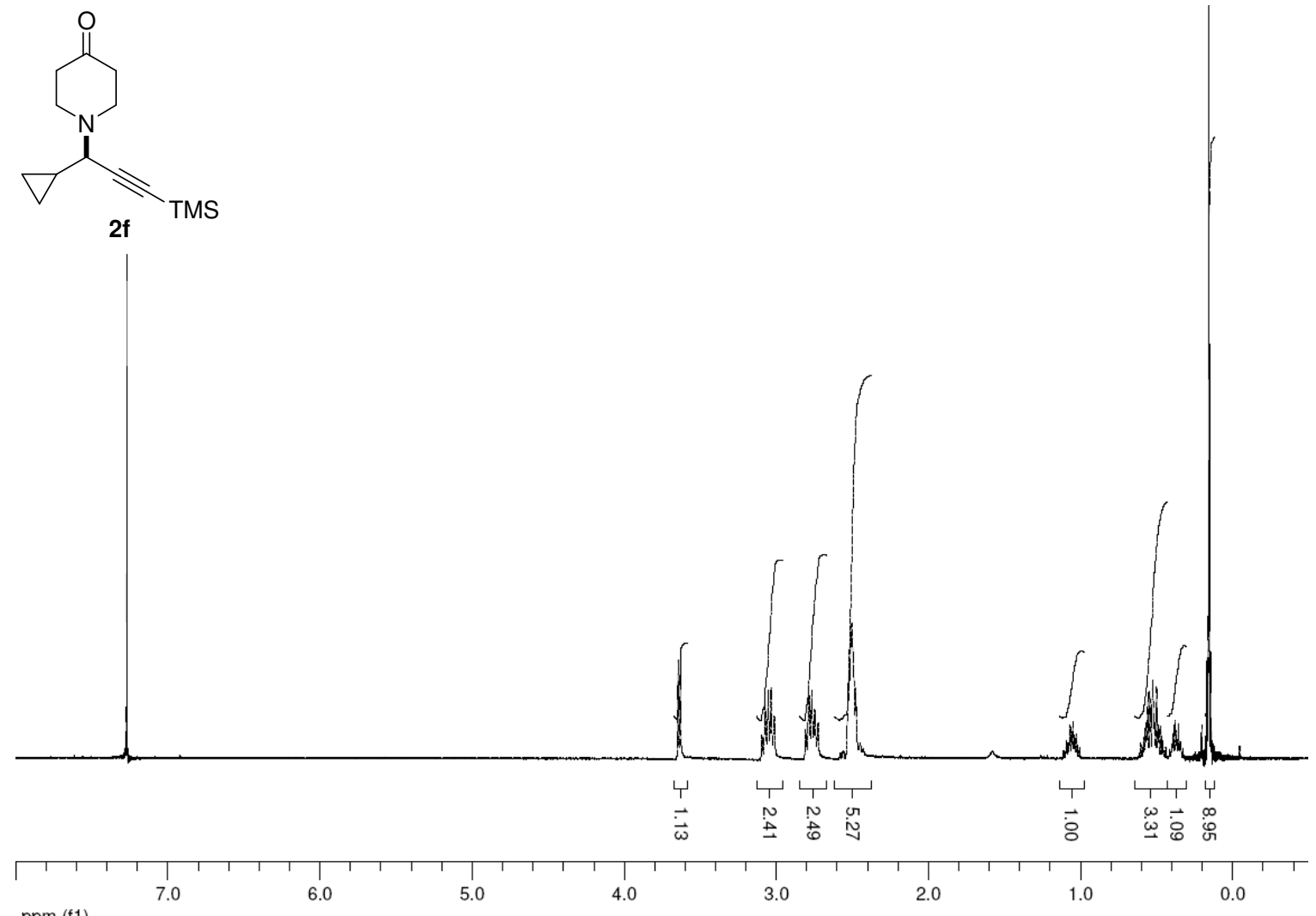
ppm (f1)
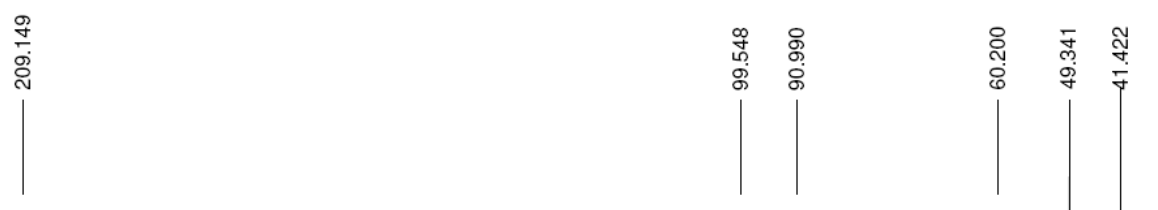

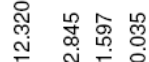

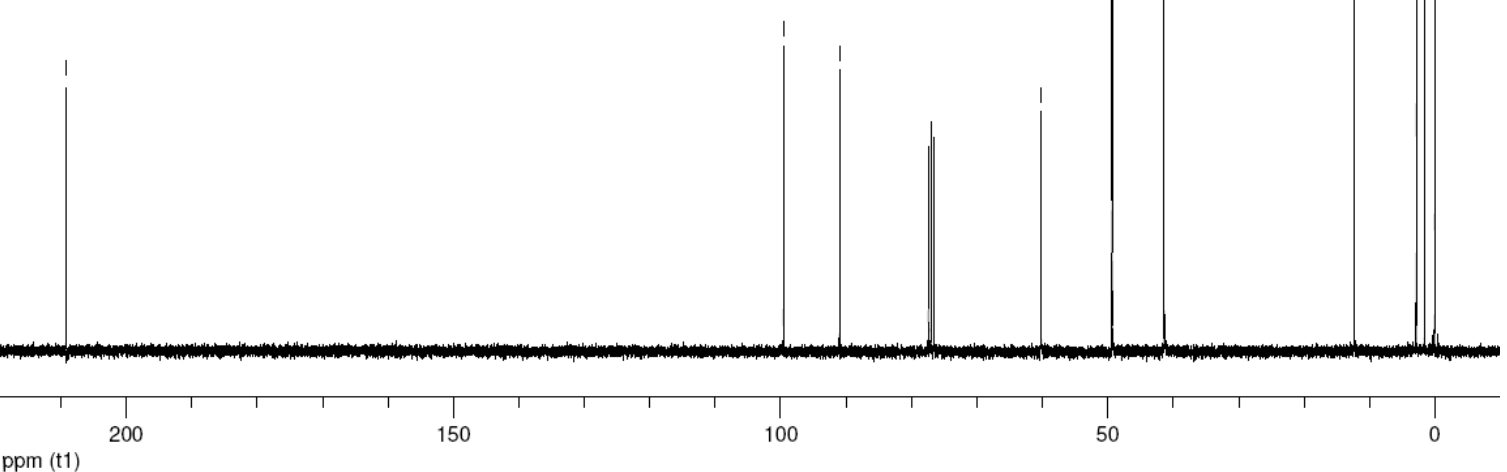



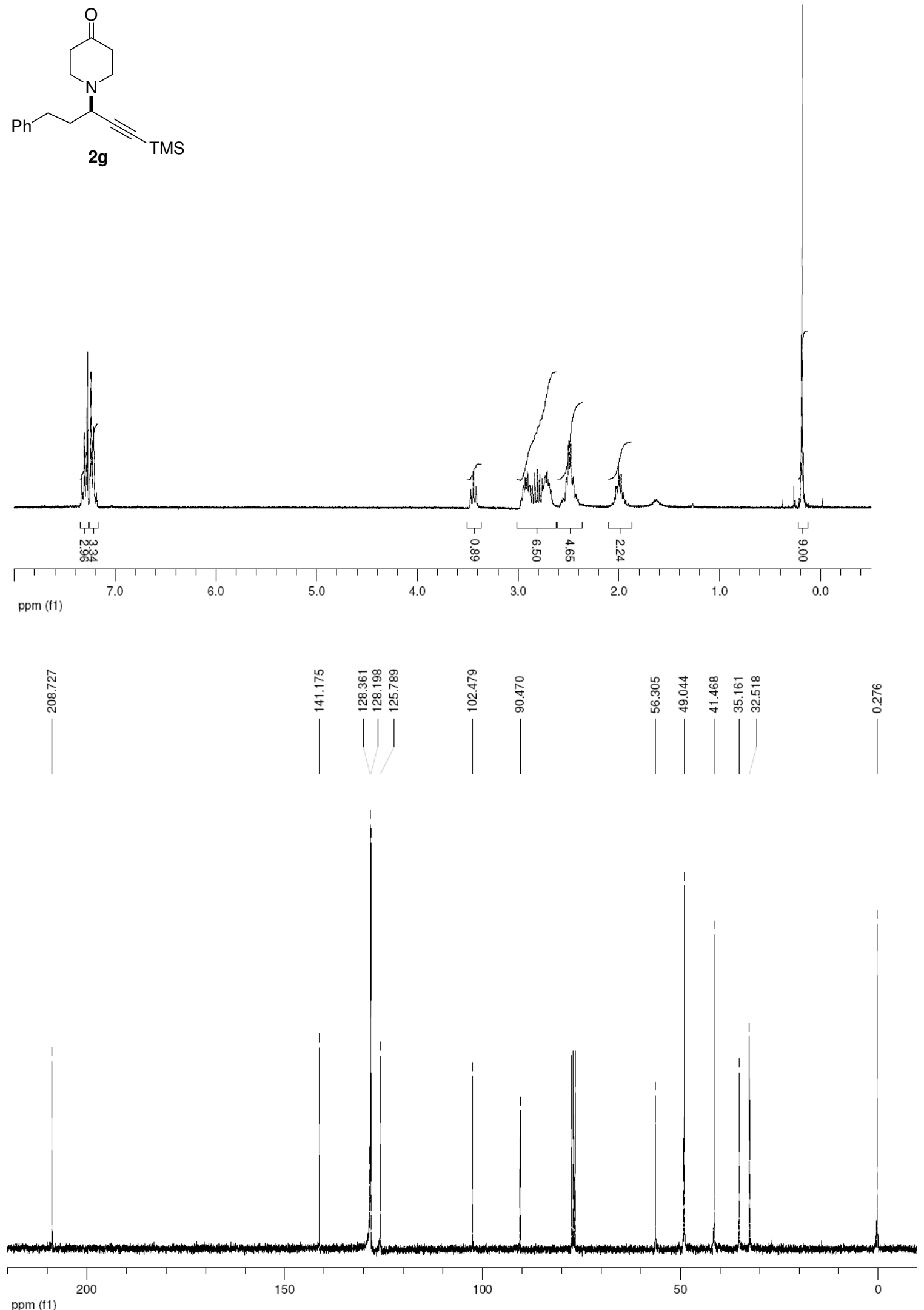


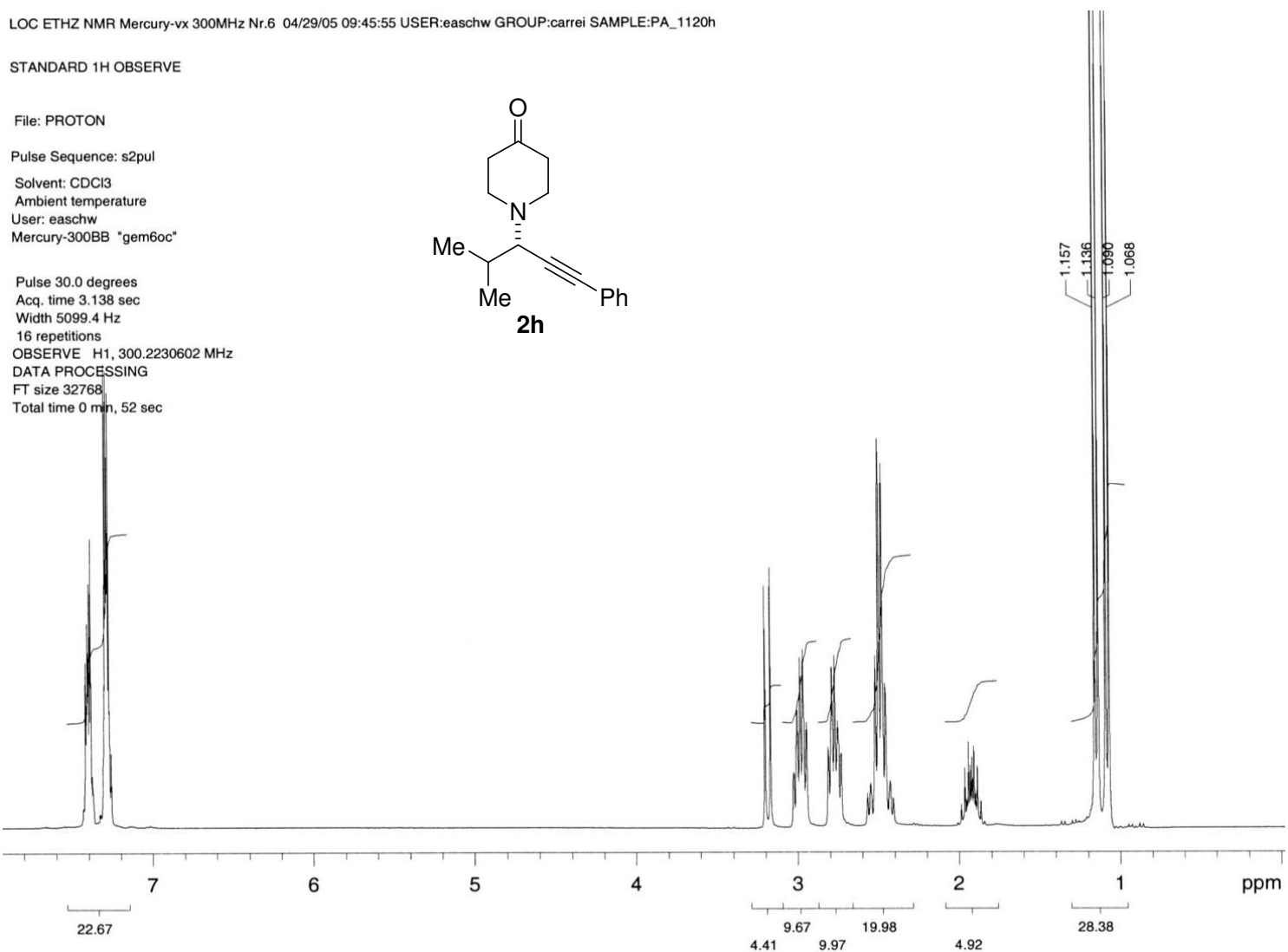

LOC ETHZ NMR Mercury-vx 300MHz Nr.6 04/29/05 09:56:44 USER:easchw GROUP:carrei SAMPLE:PA_1120c

13C OBSERVE

File: CARBON

Pulse Sequence: s2pul

Solvent: $\mathrm{CDCl} 3$

Ambient temperature

User: easchw

Mercury-300BB "gem6oc"

Relax. delay $1.000 \mathrm{sec}$

Pulse 45.0 degrees

Acq. time $1.300 \mathrm{sec}$

Width $20000.0 \mathrm{~Hz}$

256 repetitions

OBSERVE C13, $75.4911544 \mathrm{MH}$

DECOUPLE H1, 300.2242455 MHz

Power $35 \mathrm{~dB}$

continuously on

WALTZ-16 modulated

DATA PROCESSING

Line broadening $2.0 \mathrm{~Hz}$

Total time $12 \mathrm{~min}, 41 \mathrm{sec}$

$4.41^{9.67} 9.97 \quad 4.98$

m

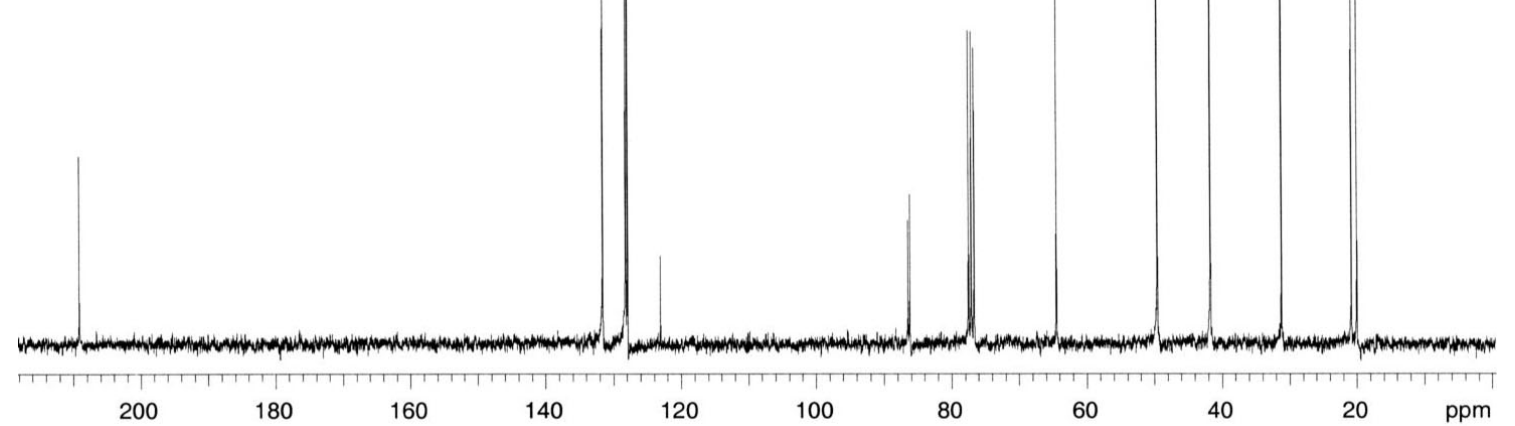


LOC ETHZ NMR Mercury-vx 300MHz Nr.6 05/24/05 16:37:19 USER:easchw GROUP:carrei SAMPLE:PA_1126h

STANDARD 1 H OBSERVE

File: PROTON

Pulse Sequence: s2pul

Solvent: CDCl3

Ambient temperature

User: easchw

Mercury-300BB "gem6oc"

Pulse 30.0 degrees

Acq. time $3.138 \mathrm{sec}$

16 repetitions

OBSERVE H1, 300.2230602 MHz

DATA PROCESSING

FT size 32768

Total time $0 \mathrm{~min}, 52 \mathrm{sec}$

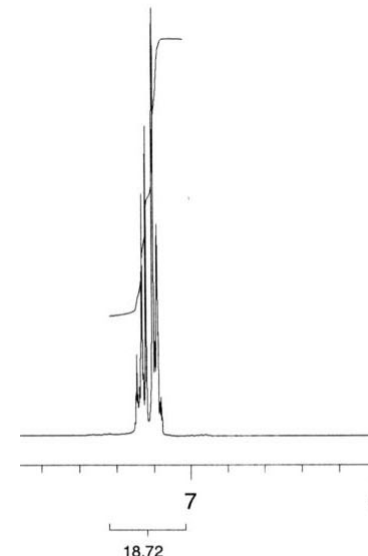

18.72

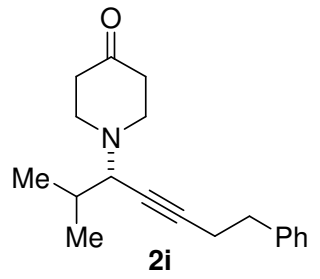

2

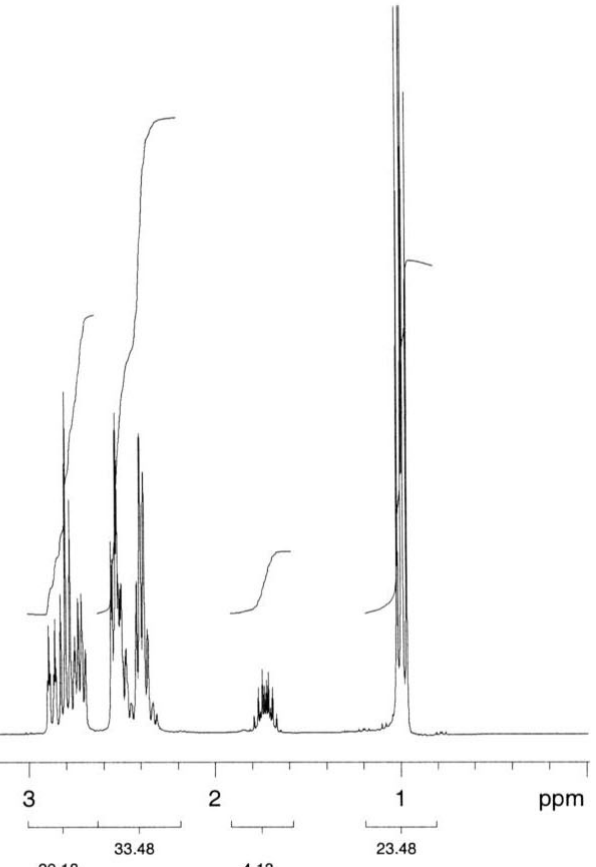

LOC ETHZ NMR Mercury-vx 300MHz Nr.6 05/24/05 16:47:14 USER:easchw GROUP:carrei SAMPLE:PA_1126c

13C OBSERVE

File: CARBON

Pulse Sequence: s2pul

Solvent: $\mathrm{CDCl} 3$

Ambient temperature

Mercury-300BB "gem6oc"

Relax. delay $1.000 \mathrm{sec}$

Pulse 45.0 degrees

Acq. time $1.300 \mathrm{sec}$

Width $20000.0 \mathrm{~Hz}$

OBSERVE C13, $75.4911544 \mathrm{MHz}$

DECOUPIE H1, 3002242455 MHz

Power $35 \mathrm{~dB}$

Continuously on

DATA PROCESSING

Line broadening $2.0 \mathrm{~Hz}$

FT size 65536

Total time $21 \mathrm{~min}, 11 \mathrm{sec}$

तू

ฉे

$6 \quad 5$

T $1+1+1$
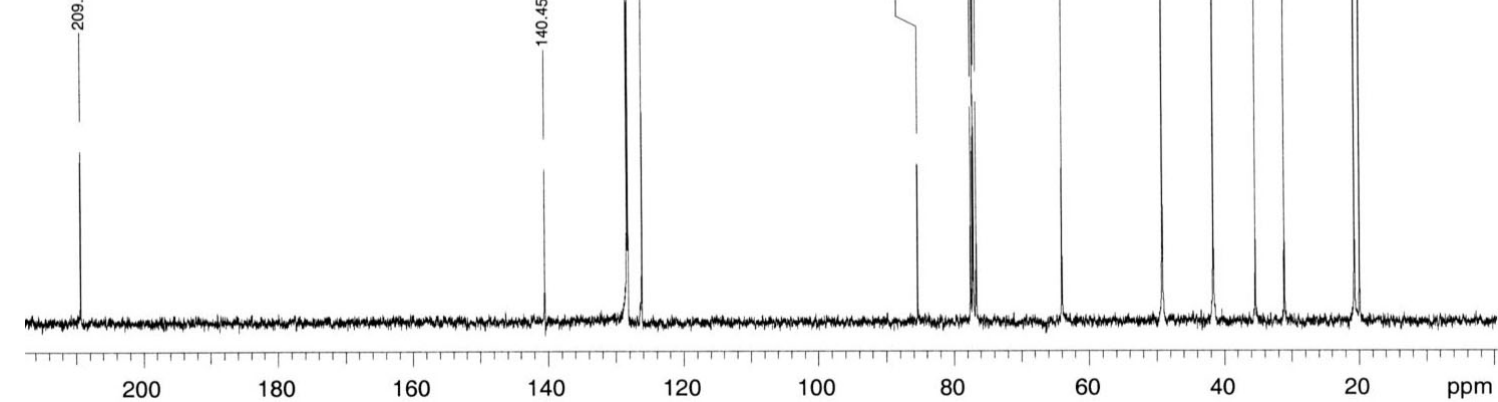
LOC ETHZ NMR Mercury-vx 300MHz Nr.6 06/02/05 16:47:08 USER:easchw GROUP:carrei SAMPLE:PA_1132.3h

STANDARD 1 H OBSERVE

File: PROTON

Pulse Sequence: s2pul

Solvent: $\mathrm{CDCl} 3$

Ambient temperature

Mercury-300BB "gem6oc"

Pulse 30.0 degrees

Acq. time $3.138 \mathrm{sec}$

Width $5099.4 \mathrm{H}$
16 repetitions

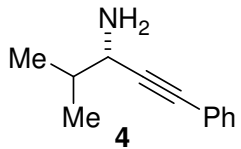

VE $\mathrm{H} 1,300.2230602 \mathrm{MHz}$

DATA PROCESSING

Total time $0 \mathrm{~min}, 52 \mathrm{sec}$

$52 \mathrm{sec}$

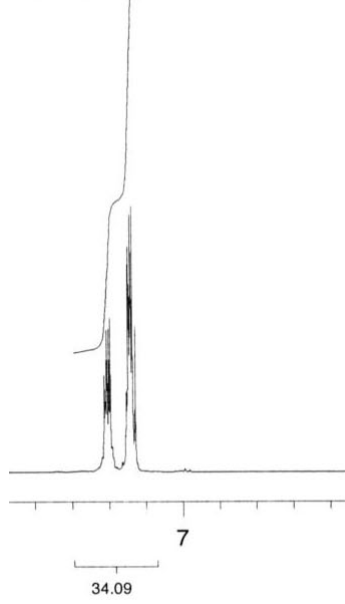

34.09

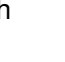



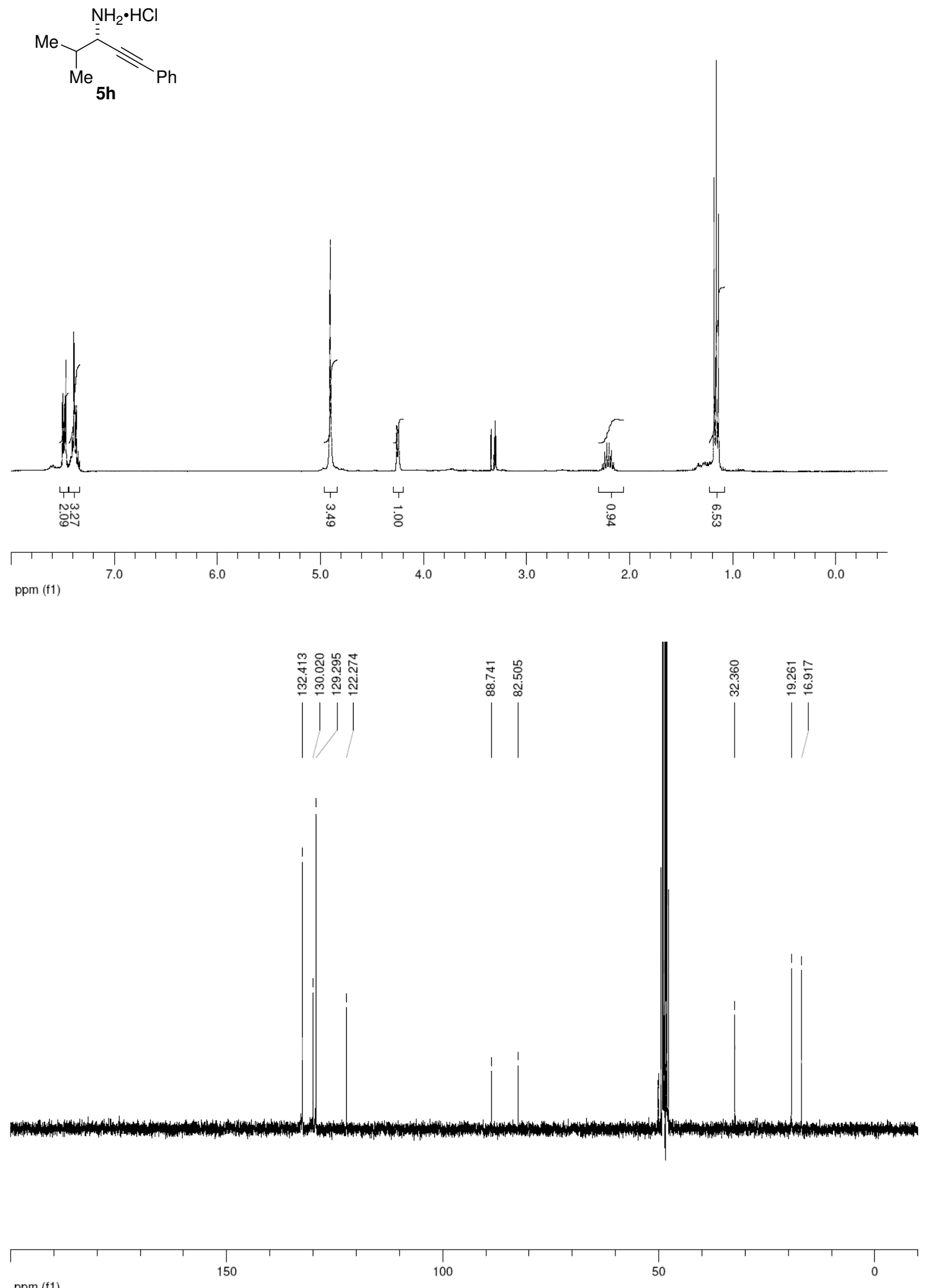

ppm (f1) 

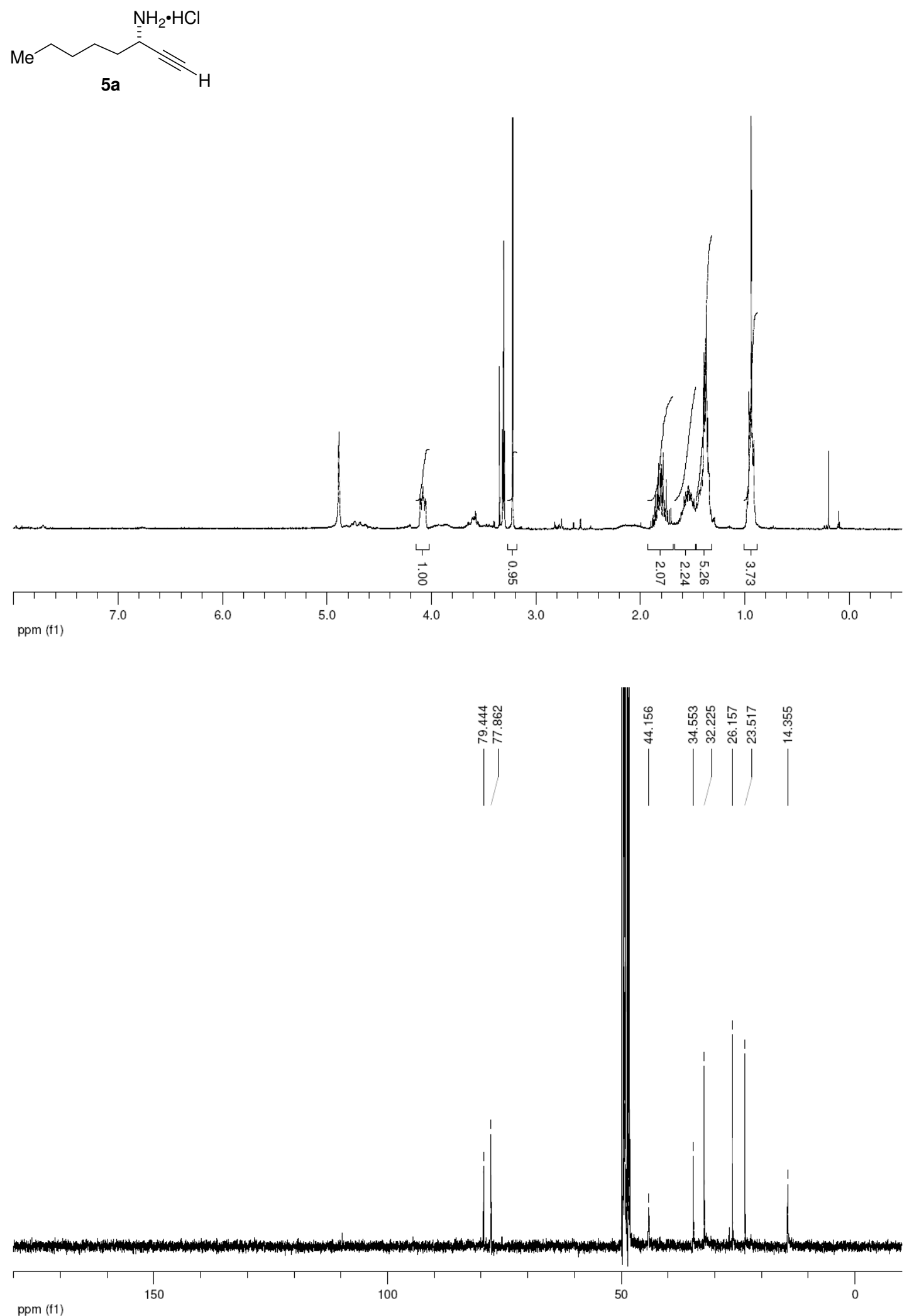

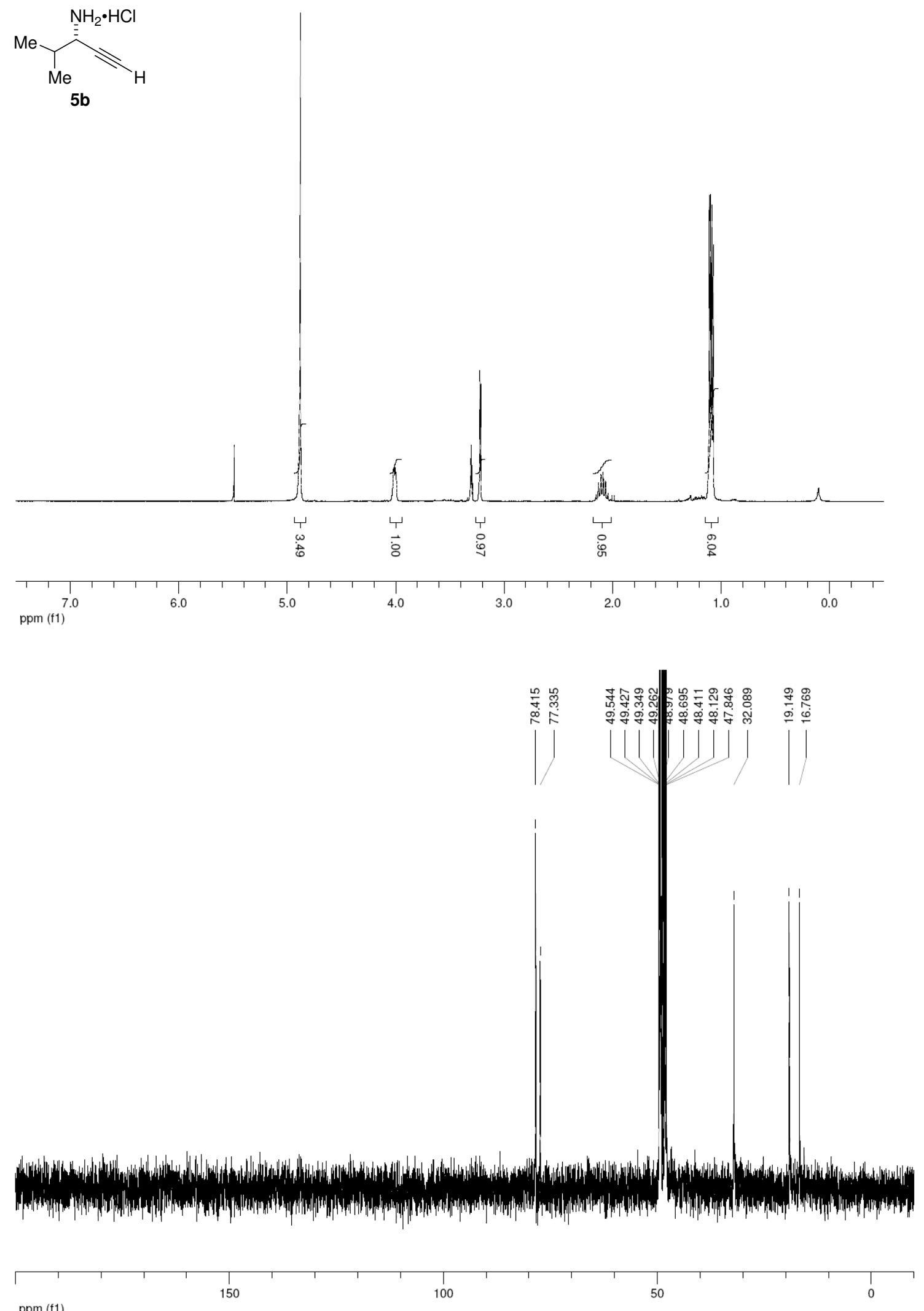

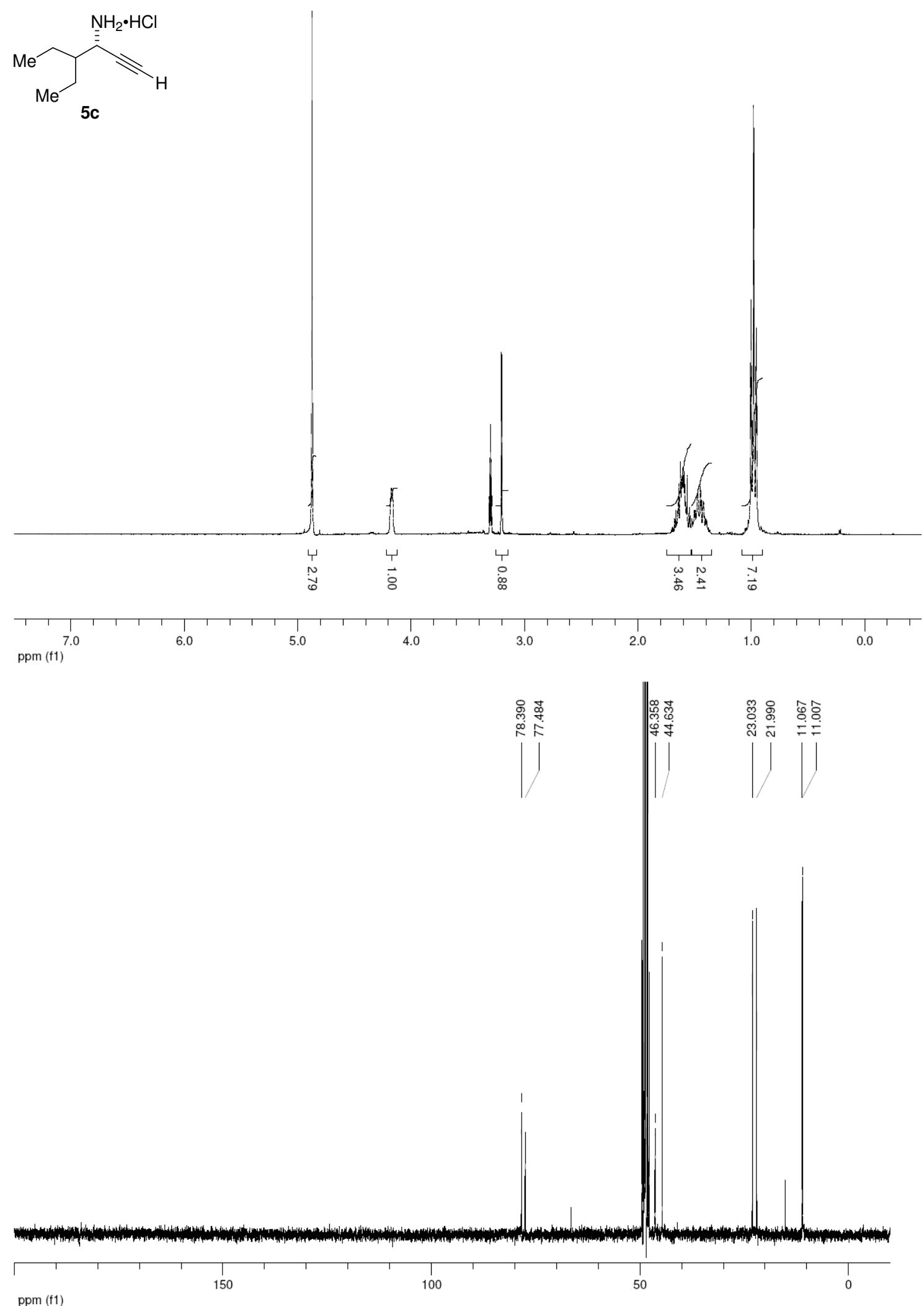

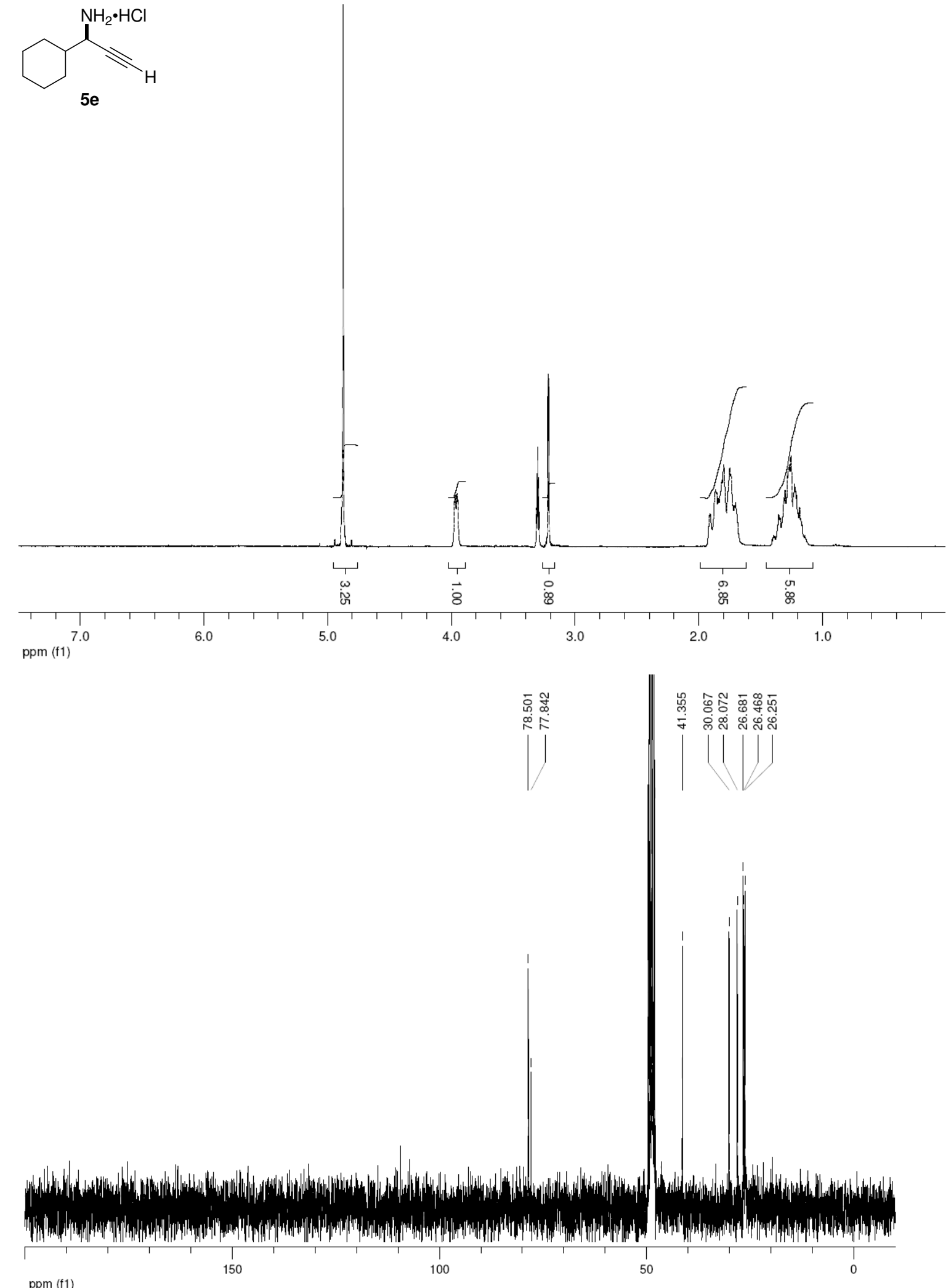

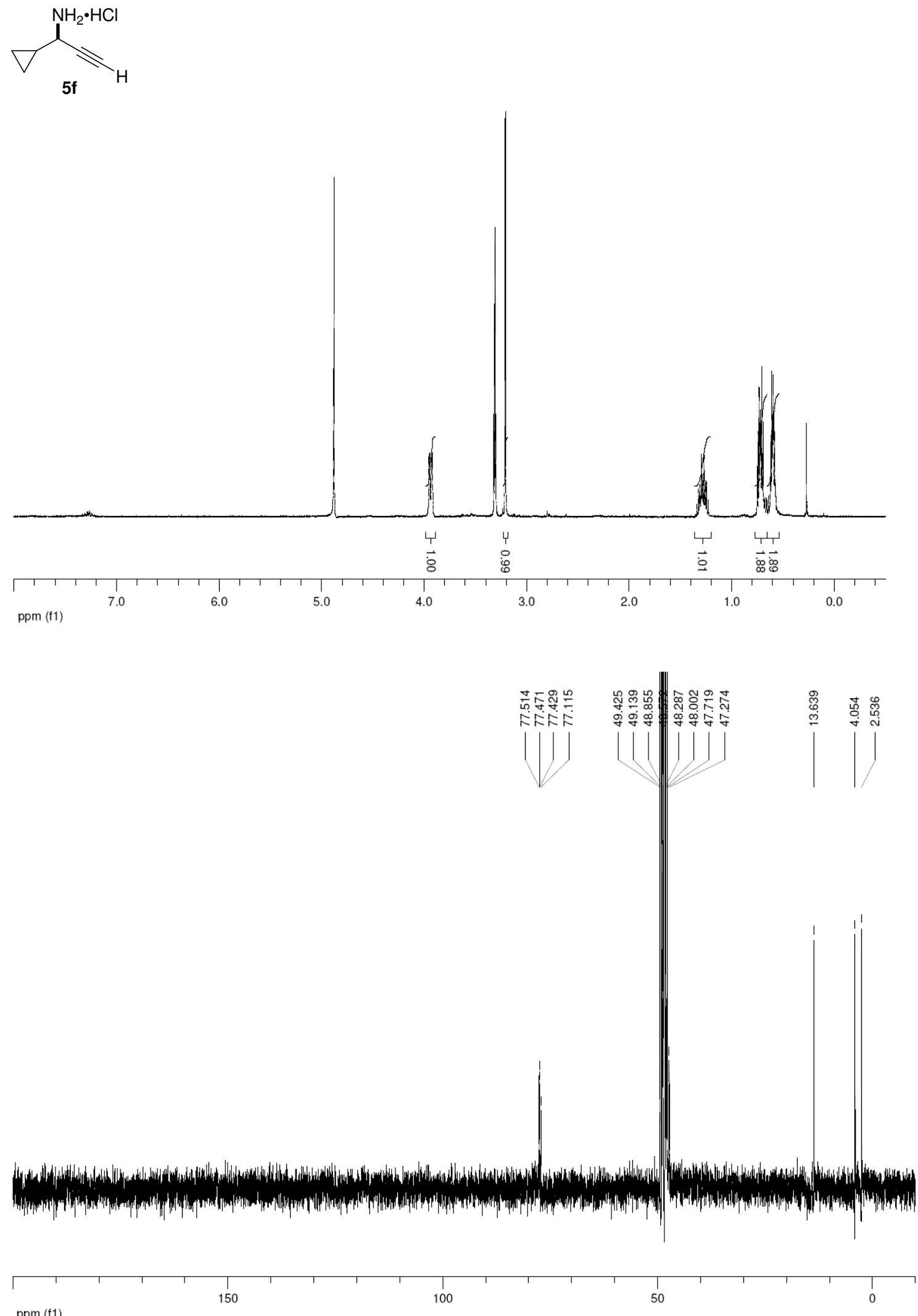

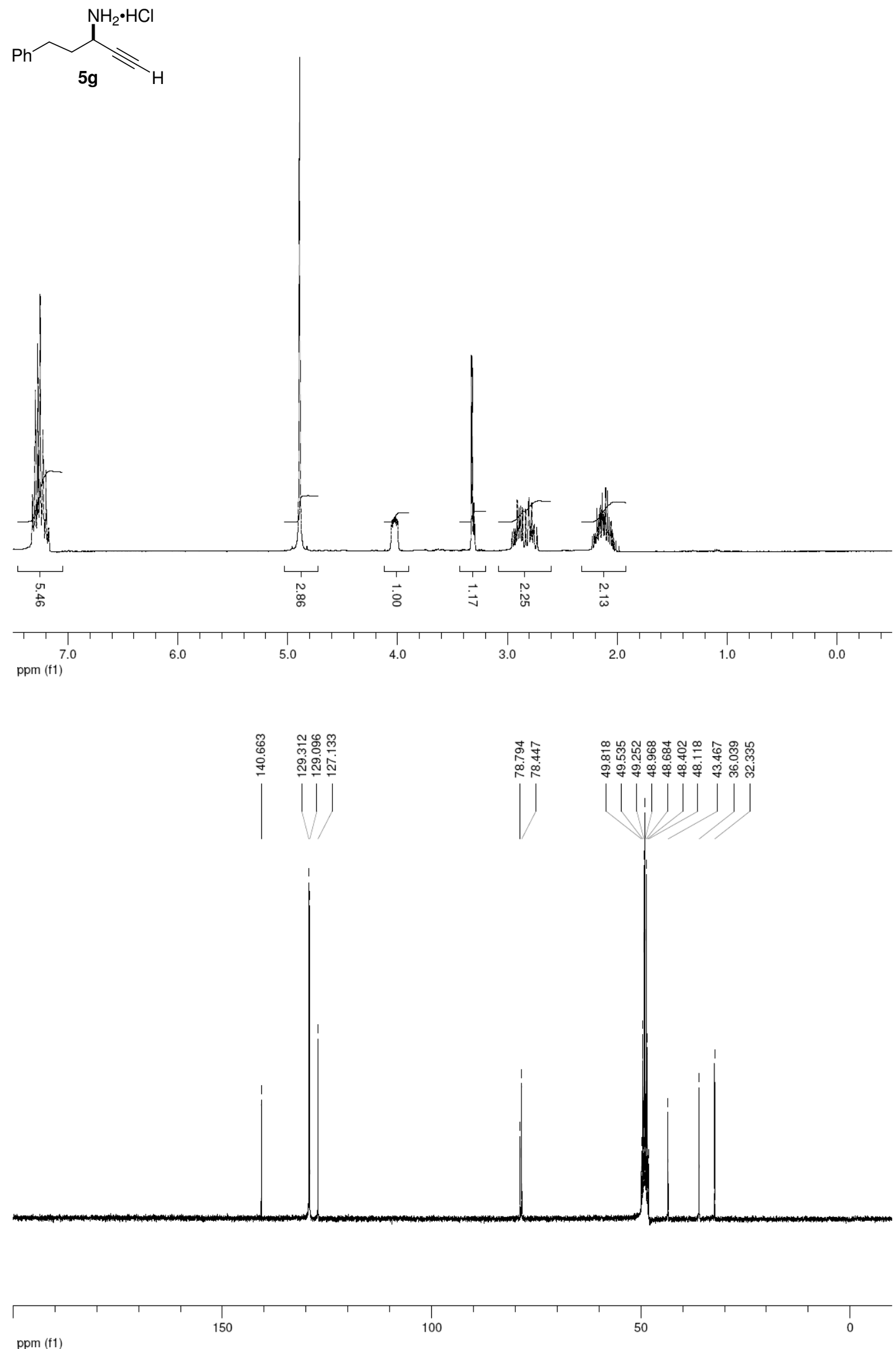